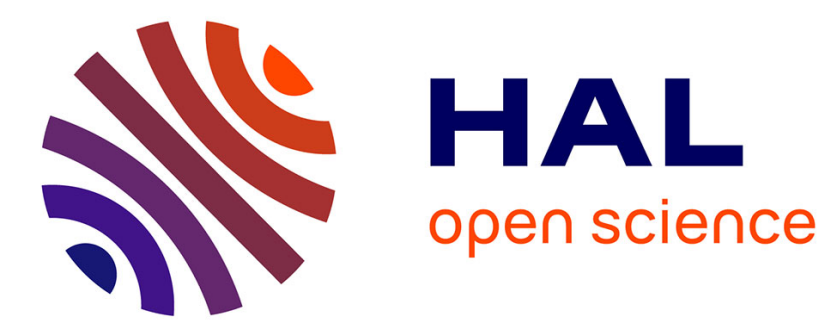

\title{
On Super-High-Frequencies in Discontinuous 1st-Order Delay-Differential Equations
}

\author{
Marianne Akian, Pierre-Alexandre Bliman
}

\section{To cite this version:}

Marianne Akian, Pierre-Alexandre Bliman. On Super-High-Frequencies in Discontinuous 1st-Order Delay-Differential Equations. [Research Report] RR-3443, INRIA. 1998. inria-00077201

\section{HAL Id: inria-00077201 \\ https://hal.inria.fr/inria-00077201}

Submitted on 29 May 2006

HAL is a multi-disciplinary open access archive for the deposit and dissemination of scientific research documents, whether they are published or not. The documents may come from teaching and research institutions in France or abroad, or from public or private research centers.
L'archive ouverte pluridisciplinaire HAL, est destinée au dépôt et à la diffusion de documents scientifiques de niveau recherche, publiés ou non, émanant des établissements d'enseignement et de recherche français ou étrangers, des laboratoires publics ou privés. 
INSTITUT NATIONAL DE RECHERCHE EN INFORMATIQUE ET EN AUTOMATIQUE

\title{
On super-high-frequencies in discontinuous 1st-order delay-differential equations
}

\author{
M. Akian, P.-A. Bliman
}

\section{$\mathbf{N}^{\circ} 3443$}

June 22, 1998

THÈME 4 



\title{
RINRIA
}

\section{On super-high-frequencies in discontinuous 1st-order delay-differential equations}

\author{
M. Akian* , P.-A. Bliman ${ }^{\dagger}$ \\ Thème 4 - Simulation et optimisation \\ de systèmes complexes \\ Projets Sosso et Meta2 \\ Rapport de recherche $\mathrm{n}^{\circ} 3443$ - June 22,1998 - 32 pages
}

\begin{abstract}
One considers a scalar 1st-order nonlinear differential equation with a delayed relay-output proportional feedback. One shows that, under a boundedness condition on the nonlinearity only, any solution of this equation has, after a finite time, a finite number of zeros on compact sets. An estimate of the time after which the super-high-frequency disappears is provided. This improves some previous works by Shustin. As a consequence, using some works by Fridman et al., any solution of the system under study coincides, after a finite time, with one of the periodic solutions.
\end{abstract}

Key-words: super-high-frequency oscillations, delay differential equations, periodic solutions, slowly oscillating solutions, relay nonlinearity.

(Résumé : tsvp)

* email: Marianne.Akian@inria.fr

$\dagger$ email: Pierre-Alexandre.Bliman@inria.fr 


\section{Sur les oscillations à super-haute fréquence d'équations différentielles à retard du 1er ordre discontinues}

Résumé : On considère une équation différentielle non-linéaire du 1er ordre avec retard et relais. On montre que, sous une condition de bornitude de la non-linéarité uniquement, toute solution de cette équation admet, au bout d'un temps fini, une nombre fini de zéros sur tout ensemble compact. On fournit de plus une estimation de la durée au bout de laquelle les super-hautes-fréquences disparaissent. On améliore ainsi des résultats obtenus précédemment par Shustin. Ceci permet de prouver, à partir de travaux de Fridman et al., que toute solution du système étudié coïncide au bout d'un temps fini avec une des solutions périodiques.

Mots-clé : oscillations à super-haute fréquence, équations différentielles à retard, solutions périodiques, solutions lentement oscillantes, relais. 


\section{Introduction}

One studies here the scalar delay-differential equation

$$
\dot{X}=-\operatorname{sgn} X(t-h)+F(X(t)), X_{0}=x_{0},
$$

where $h>0, x_{0} \in \mathcal{C}((-h, 0])$ and $\left.X_{t} \triangleq X\right|_{(t-h, t]}, t \geq 0$. This equation arises e.g. when controlling the nonlinear system $\dot{x}=F(x)+u$ by use of a proportional (negative) feedback $u$ on the delayed output of a relay sensor. For example, the case where $F$ is linear decreasing comes from an automotive control problem, see Franklin et al. [5], Akian et al. [1]. Equation (1) is a particular case of a more general class, given by

$$
\dot{X}=f(X(t), X(t-h)),
$$

which arises in a variety of models in the literature, see Diekmann et al. [3].

For equation (1), it is shown ${ }^{1}$ in Fridman et al. [6] that, under the condition:

$$
F \text { is a } \mathcal{C}^{1} \text { function such that } \sup _{x}|F(x)|<1,
$$

if $x_{0}$ has a finite number of zeros, then the same holds for $X_{t}, t \geq 0$. Indeed, denoting in this case $Z \subset[0,+\infty)$ the set of zeros of $X$ with change of sign and $\nu(t)=\operatorname{card} Z \cap\left[t^{\prime}-h, t^{\prime}\right)$ where $t^{\prime}=\inf [t,+\infty) \cap Z$, the function ${ }^{2} \nu$ is well-defined, nonincreasing and even on $[h,+\infty$ ) (see however the precise definition of $Z$ in the general case in Section 4 below). For any $n \in \mathbb{N}$, there exists a unique periodic solution with $\nu \equiv 2 n$ [6]. The slowest one only (corresponding to $n=0$ ) is orbitally stable. It is also the only slowly-oscillating ${ }^{3}$ periodic solution. Any solution $X$ departing from an initial condition $x_{0}$ with finite number of zeros, is equal, after a finite time, to one of the periodic solutions [6].

Related results have been obtained for various, mostly smooth, systems of type (2), on existence, uniqueness and stability of slowly-oscillating periodic solutions. Mallet-Paret has shown in [11] that, under conditions involving in particular a negative feedback condition in the delay, the dynamical system described by (2) possesses a global integer valued Lyapunov function (just as $\nu$ here), which gives rise to a Morse decomposition of the attractor into a finite ordered collection of compact subsets of the phase space, invariant by the flow. Each one of these subsets, called the Morse sets, contains a periodic solution [11, Theorem D]. In the case of equation (1), the Morse sets are the singletons containing the periodic trajectories with $\nu \equiv 2 n, n \in \mathbb{N}$. Due to the discontinuity of sgn on 0 , their number is infinite.

Also, results on existence of chaotic motions have been obtained by several authors. For an overview of these results and a commented bibliography, the reader is refered to

\footnotetext{
${ }^{1}$ The fields of application of the results by Fridman et al. [6] and Shustin [17] are indeed wider, as they also include some nonautonomous systems.

${ }^{2}$ Here we choose a definition of $\nu$ in the spirit of Mallet-Paret [11], whereas Shustin's choice [17] $t^{\prime}=$ $\sup [0, t] \cap Z$ necessitates special care for initialization.

${ }^{3}$ that is, such that any two distinct zeros are distant from at least the value $h$ of the delay, see Nussbaum [14], Diekmann et al. [3].
}

$\mathrm{RR} \mathrm{n}^{\circ} 3443$ 
the monography by Diekmann et al. [3, Chapters XV and XVI]. See also the papers by Peters [15] and Ivanov et al. [10] for some results concerning systems of type (2) where $f$ is discontinuous wrt the second variable.

At last, some partial results for 2nd order systems have been obtained by An der Heiden et al. [2] and Fridman et al. [7].

One is interested here by the case where $x_{0}$ is a continuous function with infinite number of zeros (super-high-frequency). In Shustin [17, Theorem 0.5], it is shown that there exists $C>0$ (dependent on $F$, but not on $x_{0}$ nor $h$ ) such that $\nu(t)$ is well-defined and finite for

$$
t \geq h C\left(1+\left(\frac{\delta_{0}}{h}\right)^{-4}\right) \text { with } \delta_{0} \triangleq \max \left\{t-t^{\prime}:-h \leq t^{\prime} \leq t \leq 0, x_{0} \neq 0 \text { on }\left(t^{\prime}, t\right)\right\} .
$$

However, together with (3), a supplementary condition is required in Shustin [17, Formula (0.6)], namely that $(F(h x)-F(0)) / x$ be small, together with its first derivative. This can be interpreted as a small-delay condition. In Theorem 2, one generalizes Shustin's result and sharpen (4). For the proof, one uses the technique of Shustin [17], that is, for a solution $X$ of (1), one considers a "measure" on the lengths of open intervals where $X_{t}$ has constant sign and proves that this measure strictly decreases with respect to $n$, when $t=\alpha-n h$ and $\alpha$ is an accumulation point of the set $Z$ of zeros of $X$ with change of sign. The measure being bounded, this leads to a bound on $\alpha$, and then on $t$ such that $\nu(t)$ is infinite. In short, one proves that the super-high-frequencies are asymptotically stable for the backward time flow, and then unstable for the forward time flow. Such a technique is also used in Fridman et al. [6] and Akian et al. [1] to prove the unstability of the non slowly-oscillating periodic solutions.

Some results of Fridman et al. [6] and Shustin [17] (namely the fact that $\nu$ decreases and the disappearance of super-high-frequency oscillations) have been generalized by Dix [4] in the case of a varying delay such that $\mathrm{Id}-h$ is strictly increasing. One believes that the results presented in the present paper may be generalized in the same way.

An important point in equation (1) is the choice of sgn0. Here, sgn is chosen as a singlevalued map, otherwise uniqueness of solution of (1) cannot be expected, due to the delay. Instead of choosing sgn0 $=0$ as in Shustin [17], it seems more realistic, in the context of control, to take $\operatorname{sgn} 0 \in\{-1,+1\}$ : the sign usually models a binary sensor or actuator. Let us define finally $\operatorname{sgn} X$ as follows. Let $z \in L^{\infty}(-h,+\infty)$ with $|z(t)| \equiv 1$, one denotes:

$$
\operatorname{sgn} X(t)= \begin{cases}1 & \text { if } X(t)>0 \\ -1 & \text { if } X(t)<0 \\ z(t) & \text { if } X(t)=0\end{cases}
$$

This permits to model various policies: e.g. $\operatorname{sgn} 0=1$, or $\operatorname{sgn} X(t)$ switches as late as possible ... An important consequence is that any solution of (1)-(5) verifies: meas $\{t \geq 0: X(t)=$ $0\}=0$, see Lemma 5. Moreover, as solving (1) requires regularity of the right-hand side 
only in those points where it vanishes, (5) permits to weaken the regularity assumption on $F$.

The main results are given in Section 2. Theorem 1 states the existence of solutions of (1)-(5), proved in Section 3. Theorem 2 states the disapperance of super-high-frequency oscillations, proved in Section 4. In Corollary 3, the condition $\sup _{x}|F(x)|<1$ is weakened. In Corollary 4, boundedness of the solutions of (1)-(5) is proved using Theorem 2 and the results of Fridman et al. [6]. The bounds given there are optimal.

\section{Main results}

Let us first state the existence of solutions to (1).

Theorem 1. Suppose that $F$ is a measurable function such that $\sup _{x \in \mathbb{R}}|F(x)|<1$. Then, for any $x_{0} \in \mathcal{C}((-h, 0])$, there exists at least one function $X \in \mathcal{C}((-h,+\infty))$, absolutely continuous on $[0,+\infty)$ and satisfying (1)-(5) almost everywhere. By definition, such a function is called a solution of (1)-(5) on $[0,+\infty)$.

Uniqueness of the solution is guaranteed when $x_{0}$ has a finite number of zeros, see Section 3. For general initial conditions, it may be ensured by assuming e.g. $F$ Lispchitz continuous or $F$ nonincreasing. However, there is no evidence that the measurability of $F$ does not guarantee uniqueness too.

Our central result is the following:

Theorem 2. Suppose that $F$ is a measurable function such that $\sup _{x \in \mathbb{R}}|F(x)|<1$. Then, for any $x_{0} \in \mathcal{C}((-h, 0])$, there exists $t_{x_{0}} \geq 0$ such that, for any solution $X$ of (1)-(5) on $[0,+\infty), X_{t}$ has a finite number of zeros for $t \geq t_{x_{0}}$. For any $\varepsilon>0$, there exists $C_{\varepsilon}>0$ (dependent on $\sup _{x}|F(x)|$ only), such that

$$
\begin{aligned}
t_{x_{0}} \leq h C_{\varepsilon} & \left(1+\left(\frac{\delta}{h}\right)^{-2-\varepsilon}\right) \\
& \text { with } \delta \triangleq \sup \left\{\text { meas } I: I \text { interval } \subset(-h, 0), \text { sgn } x_{0} \text { constant a.e. on } I\right\} .
\end{aligned}
$$

The value of $\delta$ depends jointly upon $x_{0}$ and $z$, and one shows easily that $\delta \geq \delta_{0}$.

Corollary 3. Suppose that $F$ is measurable, and that there exists an open set $\Omega \subset \mathbb{R}$ such that $\sup _{x \in \Omega}|F(x)|<1$ and such that, for any solution $X$ of (1)-(5) on $[0,+\infty)$, there exists $t_{X, 0}>0$ with $X(t) \in \Omega$ for $t \geq t_{X, 0}$. Then, for any solution $X$ of (1)-(5) on $[0,+\infty)$, there exists $t_{X}>0$ such that $X_{t}$ has a finite number of zeros for $t>t_{X}$.

The assumptions of Corollary 3 are fulfilled e.g. when $F$ verifies: $x F(x) \leq 0$ on $\mathbb{R}$. In particular, the case treated in Akian et al. [1], where $F(x) \equiv-x$, follows from Corollary 3. Proof of Corollary 3 is left to the reader.

$\mathrm{RR} \mathrm{n}^{\circ} 3443$ 
When no super-high-frequency may be sustained, the asymptotic behavior is summarized by the periodic solutions. This offers the possibility to express some asymptotic properties of the solutions. As an example, one may prove the estimates contained in the following corollary, which are the best possible. Let us define the increasing functions $\mathcal{F}_{ \pm}$by

$$
\mathcal{F}_{ \pm}(b) \triangleq \int_{0}^{b} \frac{d x}{1 \pm F(x)} .
$$

Corollary 4. If the assumptions of Corollary 3 are fulfilled, then, for any solution $X$ of (1)-(5) on $[0,+\infty)$, there exists $t_{X} \geq 0$ such that, for any $t \geq t_{X}, t \mapsto X(t)$ is periodic, and

$$
\begin{gathered}
\left(\mathcal{F}_{-}\right)^{-1}(-h) \leq X(t) \leq\left(\mathcal{F}_{+}\right)^{-1}(h) \\
\sup \left\{t^{\prime}-t: t^{\prime} \geq t, X \geq 0 \text { a.e. on }\left[t, t^{\prime}\right]\right\} \leq T_{+} \triangleq h+\mathcal{F}_{-} \circ\left(\mathcal{F}_{+}\right)^{-1}(h), \\
\sup \left\{t^{\prime}-t: t^{\prime} \geq t, X \leq 0 \text { a.e. on }\left[t, t^{\prime}\right]\right\} \leq T_{-} \triangleq h-\mathcal{F}_{+} \circ\left(\mathcal{F}_{-}\right)^{-1}(-h) .
\end{gathered}
$$

Moreover, if $F$ is odd, then, for any solution $X$ of (1)-(5) on $[0,+\infty)$,

$$
\lim _{t \rightarrow+\infty} \frac{1}{t} \int_{0}^{t} X(t) d t=0 \text {. }
$$

The bounds on $X$ in (8) are null when $h$ vanishes. This must be linked with the fact that when $h=0$, any solution of (1)-(5) vanishes identically after a finite time. The inequalities in $(9),(10)$ are optimal for the slowly-oscillating periodic solution; the period of this latter is equal to $T_{+}+T_{-}$with $T_{ \pm}$defined in (9), (10).

Proof of Corollary 4. When $F$ is regular, Fridman et al. [6] proved that, when the initial condition $x_{0}$ has a finite number of zeros, any solution of (1)-(5) is equal, after a finite time $t_{x_{0}}$, to one of the periodic solutions. Using Theorem 2, the restriction on the number of zeros may be removed. Now, it is easy to show that the amplitude and the intervals of constant sign of the slowly-oscillating solution are larger than the same quantities computed for the other periodic solutions. Using this property, one gets (8) to (10). Indeed, for $t \geq t_{x_{0}}$, one gets

$$
\left.\sup \left\{t^{\prime}-t: t^{\prime} \geq t, X \geq 0(\text { resp. } X \leq 0) \text { a.e. on }\left[t, t^{\prime}\right]\right\} \leq T_{+} \text {(resp. } T_{-}\right),
$$

where $T_{+}$(resp. $T_{-}$), defined by (9) (resp. (10)), is the length of the time intervals on which the slowly-oscillating solution is positive (resp. negative), see the beginning of Section 4.2.1 below. One then deduces that

$$
\left(\mathcal{F}_{+}+\mathcal{F}_{-}\right)^{-1}\left(-T_{-}\right) \leq X(t) \leq\left(\mathcal{F}_{+}+\mathcal{F}_{-}\right)^{-1}\left(T_{+}\right),
$$

see Formula (23) and its interpretation. 
When $F$ is odd, then $T_{+}=T_{-}$, and the other periodic solutions have the same symmetry property. More precisely, for any periodic solution $X^{*}$ of least period $T>0$, for any $t \in[0,+\infty)$, one has

$$
X^{*}\left(t+\frac{T}{2}\right)=-X^{*}(t) .
$$

Formula (11) is deduced from this property.

When $F$ is non regular, this proof may be generalized by adapting the proof by Fridman et al. [6].

\section{Proof of Theorem 1 (Existence result)}

Let us prove the existence of a solution of $(1)-(5)$ on $[0, h]$. The global existence result is then obtained by induction. Let us denote

$$
M \triangleq\left\{t \in(0, h): \operatorname{sgn} x_{0}(t-h)=-1\right\} .
$$

Since $M$ is measurable, there exists a nonincreasing sequence of open sets $U_{n}$ of $(0, h)$ such that

$$
M \subset U_{n}, \quad \operatorname{meas}\left(\bigcap_{n=1}^{+\infty} U_{n} \backslash M\right)=0 .
$$

As equation (1)-(5) has to be verified a.e., one may suppose that $M=\bigcap_{n=1}^{+\infty} U_{n}$. Moreover, for any $n \in \mathbb{N}, U_{n}$ is a finite or countable union of disjoint intervals $\left(t_{n, m}, t^{\prime}{ }_{n, m}\right)$, and one denotes

$$
V_{n, m} \triangleq \bigcup_{m^{\prime}=1}^{m}\left(t_{n, m^{\prime}}, t_{n, m^{\prime}}^{\prime}\right)
$$

When $M=V_{n, m}$ for certain $n, m \in \mathbb{N} \backslash\{0\},(1)$-(5) admits a unique solution $X_{n, m}$ on $[0, h]$. Indeed, assuming that the intervals $\left(t_{n, m^{\prime}}, t_{n, m^{\prime}}^{\prime}\right)$ have been reordered, that is $t_{n, m^{\prime}}^{\prime} \leq$ $t_{n, m^{\prime}+1}$, and denoting $t_{n, 0}^{\prime}=0, t_{n, m+1}=h$, one has

$$
\left\{\begin{array}{l}
X_{n, m}(0)=x_{0}(0), \\
X_{n, m}(t)=\mathcal{F}_{-}^{-1}\left(\mathcal{F}_{-}\left(X_{n, m}\left(t^{\prime}{ }_{n, m^{\prime}}\right)\right)-\left(t-t^{\prime}{ }_{n, m^{\prime}}\right)\right) \text { if } t \in\left(t^{\prime}{ }_{n, m^{\prime}}, t_{n, m^{\prime}+1}\right), m^{\prime}=0, \ldots, m \\
X_{n, m}(t)=\mathcal{F}_{+}^{-1}\left(\mathcal{F}_{+}\left(X_{n, m}\left(t_{n, m^{\prime}}\right)\right)+\left(t-t_{n, m^{\prime}}\right)\right) \text { if } t \in\left(t_{n, m^{\prime}}, t^{\prime}{ }_{n, m^{\prime}}\right), m^{\prime}=1, \ldots, m,
\end{array}\right.
$$

where $\mathcal{F}_{ \pm}$are defined in (7). Denote

$$
\lambda \triangleq \frac{1-\sup _{x \in \mathbb{R}}|F(x)|}{2} \in\left(0, \frac{1}{2}\right],
$$


then

$$
\frac{1}{2}<\frac{1}{2(1-\lambda)}=\frac{1}{1+\sup |F|} \leq \mathcal{F}_{ \pm}^{\prime}(b) \leq \frac{1}{1-\sup |F|}=\frac{1}{2 \lambda} .
$$

For any $n, m \in \mathbb{N} \backslash\{0\}, X_{n, m} \in \mathcal{C}([0, h])$ and is differentiable a.e., with $\left|\dot{X}_{n, m}(t)\right| \leq 2(1-\lambda)$ a.e. Hence, by Ascoli's Theorem, there exist a subsequence also denoted $X_{n, m}$ and a function $X_{n} \in \mathcal{C}([0, h])$, such that $X_{n, m} \rightarrow X_{n}$ in $\mathcal{C}([0, h])$ when $m \rightarrow+\infty$. The limit $X_{n}$ is Lipschitz continuous with constant $2(1-\lambda)$. As well, there exist a subsequence also denoted $X_{n}$ and a function $X \in \mathcal{C}([0, h])$, such that $X_{n} \rightarrow X$ when $n \rightarrow+\infty$, and $X$ is Lipschitz continuous. Both $X_{n}$ and $X$ are absolutely continuous. To prove that $X$ is a solution of (1)-(5) on $[0, h]$ is equivalent to show that, for any $t \in[0, h]$,

$$
\begin{aligned}
t & =\int_{0}^{t} \frac{\dot{X}(s)}{-\operatorname{sgn} x_{0}(s-h)+F(X(s))} d s \\
& =\int_{[0, t] \cap M} d \mathcal{F}_{+}(X(s))-\int_{[0, t] \cap M^{\mathrm{c}}} d \mathcal{F}_{-}(X(s)) \\
& =\mathcal{F}_{-}(X(0))-\mathcal{F}_{-}(X(t))+\int_{[0, t] \cap M} d\left(\mathcal{F}_{+}+\mathcal{F}_{-}\right)(X(s)),
\end{aligned}
$$

where $M^{\mathrm{c}} \triangleq[0, h] \backslash M$.

Suppose first that $M=U_{n}$ (that is $X_{n}=X$ ) for a certain $n \in \mathbb{N} \backslash\{0\}$. Let $\varepsilon>0$, there exists $\underline{m}$ such that meas $\left(U_{n} \backslash V_{n, \underline{m}}\right) \leq \varepsilon$. One has, for $t \in[0, h]$ :

$$
t+\mathcal{F}_{-}\left(X_{n, m}(t)\right)-\mathcal{F}_{-}\left(X_{n, m}(0)\right)=\int_{[0, t] \cap V_{n, m}} d\left(\mathcal{F}_{+}+\mathcal{F}_{-}\right)\left(X_{n, m}(s)\right) .
$$

The left-hand side of the previous formula tends to $t+\mathcal{F}_{-}\left(X_{n}(t)\right)-\mathcal{F}_{-}\left(X_{n}(0)\right)$ when $m \rightarrow$ $+\infty$. Now, if $m \geq \underline{m}$, then

$$
\begin{aligned}
& \left|\int_{[0, t] \cap V_{n, m}} d\left(\mathcal{F}_{+}+\mathcal{F}_{-}\right)\left(X_{n, m}(s)\right)-\int_{[0, t] \cap U_{n}} d\left(\mathcal{F}_{+}+\mathcal{F}_{-}\right)\left(X_{n}(s)\right)\right| \\
& \leq \frac{1}{\lambda}\left(\operatorname{meas}\left(V_{n, m} \backslash V_{n, \underline{m}}\right)+\operatorname{meas}\left(U_{n} \backslash V_{n, \underline{m}}\right)\right) \\
& \quad+\left|\int_{[0, t] \cap V_{n, \underline{m}}} d\left(\mathcal{F}_{+}+\mathcal{F}_{-}\right)\left(X_{n, m}(s)\right)-d\left(\mathcal{F}_{+}+\mathcal{F}_{-}\right)\left(X_{n}(s)\right)\right| \\
& \leq \frac{2 \varepsilon}{\lambda}+\left|\int_{[0, t] \cap V_{n, \underline{m}}} d\left(\mathcal{F}_{+}+\mathcal{F}_{-}\right)\left(X_{n, m}(s)\right)-d\left(\mathcal{F}_{+}+\mathcal{F}_{-}\right)\left(X_{n}(s)\right)\right|,
\end{aligned}
$$

where the last integral goes to 0 when $m \rightarrow+\infty, \underline{m}$ fixed, due to the continuity of $\mathcal{F}_{+}, \mathcal{F}_{-}$ and the convergence of $X_{n, m}$ towards $X_{n}$. This being true for any $\varepsilon>0$, one gets

$$
t+\mathcal{F}_{-}\left(X_{n}(t)\right)-\mathcal{F}_{-}\left(X_{n}(0)\right)=\int_{[0, t] \cap U_{n}} d\left(\mathcal{F}_{+}+\mathcal{F}_{-}\right)\left(X_{n}(s)\right)
$$


The left-hand side of the last formula tends to $t+\mathcal{F}_{-}(X(t))-\mathcal{F}_{-}(X(0))$ when $n \rightarrow+\infty$. Let $\varepsilon>0$, there exist $\underline{n}, \underline{m}$ such that meas $\left(U_{\underline{n}} \backslash M\right) \leq \varepsilon$ and meas $\left(U_{\underline{n}} \backslash V_{\underline{n}}, \underline{m}\right) \leq \varepsilon$. If $n \geq \underline{n}$, then one has

$$
\begin{aligned}
& \left|\int_{[0, t] \cap U_{n}} d\left(\mathcal{F}_{+}+\mathcal{F}_{-}\right)\left(X_{n}(s)\right)-\int_{[0, t] \cap M} d\left(\mathcal{F}_{+}+\mathcal{F}_{-}\right)(X(s))\right| \\
& \leq \quad \frac{1}{\lambda}\left(\operatorname{meas}\left(U_{n} \triangle V_{\underline{n}, \underline{m}}\right)+\operatorname{meas}\left(M \triangle V_{\underline{n}, \underline{m}}\right)\right) \\
& \quad+\left|\int_{[0, t] \cap V_{\underline{n}, \underline{m}}} d\left(\mathcal{F}_{+}+\mathcal{F}_{-}\right)\left(X_{n}(s)\right)-d\left(\mathcal{F}_{+}+\mathcal{F}_{-}\right)(X(s))\right| \\
& \leq \frac{4 \varepsilon}{\lambda}+\left|\int_{[0, t] \cap V_{\underline{n}, \underline{m}}} d\left(\mathcal{F}_{+}+\mathcal{F}_{-}\right)\left(X_{n}(s)\right)-d\left(\mathcal{F}_{+}+\mathcal{F}_{-}\right)(X(s))\right|,
\end{aligned}
$$

where $A \triangle B \triangleq A \backslash B \cup B \backslash A$, and using the fact that $M \subset U_{n} \subset U_{\underline{n}}$ and $V_{\underline{n}, \underline{m}} \subset U_{\underline{n}}$. So, by the same arguments as before, $X$ satisfies (13) for any $t \in[0, h]$ and hence (1)-(5) a.e.

\section{Proof of Theorem 2 (Disappearance of the super- high-frequency oscillations)}

In order to prove Theorem 2, one follows the technique of Shustin [17], except that one considers the set $Z$ of zeros with change of sign instead of the set of all zeros. This is possible because of our choice of sgn0, which has as a consequence that the set of all zeros of $X$ on $[0,+\infty)$ is zero-measured (see Lemma 5). Also, the measure on the set of open intervals which is used is different, and the inequalities are sharper.

Let $X$ be a solution of (1)-(5) on $[0,+\infty)$. The precise definition of $Z$ is as follows:

$$
Z \triangleq\left\{t \geq 0: X(t)=0 \text { and } \forall \varepsilon>0, \exists t^{\prime} \in[t-\varepsilon, t), t^{\prime \prime} \in(t, t+\varepsilon], X\left(t^{\prime}\right) X\left(t^{\prime \prime}\right)<0\right\} .
$$

Let us denote $A c c Z$ the set of accumulation points of $Z$.

If $t \geq h$ and $Z \cap[t-h, t]$ is infinite, that is if $X_{t}$ has an infinite number of zeros with change of sign, then, by compacity, there exists at least one accumulation point $\alpha \in$ Acc $Z$ in $[t-h, t]$ (conversely, if $\alpha \in A c c Z$ and $\alpha \geq h$, then $X_{t}$ has an infinite number of zeros with change of sign for $\alpha<t<\alpha+h$ ). Hence, if Acc $Z$ is empty or bounded, then $X_{t}$ has a finite number of zeros with change of sign for $t>\max A c c Z+h$ (with $\max \emptyset=0$ ), and $X_{t}$ has a finite number of zeros for $t>\max A \operatorname{cc} Z+2 h$. Therefore, in order to prove Theorem 2 , one shall bound $\mathrm{Acc} Z$ (when it is not empty) by $h C_{\varepsilon}\left(1+(\delta / h)^{-2-\varepsilon}\right)$. 


\subsection{Properties of the zeros of the solution}

Lemma 5. The following properties are true:

$$
\begin{gathered}
\text { meas }\{t \geq 0: X(t)=0\}=0 \\
t \notin Z \Rightarrow X>0 \text { or } X<0 \text { a.e. in a neighborhood of } t .
\end{gathered}
$$

Proof. Denote $N \subset \mathbb{R}^{+}$a set of measure zero such that $X$ is differentiable and (1)-(5) is fulfilled outside $N$. For any $\varepsilon>0$, there exists an open set $U$ such that $N \subset U$ and meas $U<\varepsilon$. Denote $S \triangleq\{t \geq 0: X(t)=0\}$. It is clear that $S \backslash N$ has no accumulation point, otherwise on such a point $t$, one would have $X(t)=0, \dot{X}(t)=0$, so $|F(X(t))|=1$. As $S \backslash U \subset S \backslash N$, the set $S \backslash U$ has no accumulation point. Being closed (by the continuity of $X)$, it is then finite or countable. Hence, meas $(S \backslash U)=0$, so meas $S=$ meas $(S \cap U)<\varepsilon$ for any $\varepsilon>0$. This proves the first property. The second property is deduced from the first one and (14).

Lemma 6. The set $Z$ is closed.

Proof. Let $t_{i}$ be a sequence of elements of $Z$ converging to $t$. There exists a subsequence, also denoted $t_{i}$, which converges e.g. from below. Let $t_{i}^{\prime}<t_{i}<t_{i}^{\prime \prime}<t$ be such that $X\left(t_{i}^{\prime}\right) X\left(t_{i}^{\prime \prime}\right)<0$, together with $t_{i}^{\prime \prime}-t_{i}^{\prime} \rightarrow 0$ when $i \rightarrow+\infty$ (see (14)). Then $t_{i}^{\prime}, t_{i}^{\prime \prime}$ tend to $t$.

Let $\varepsilon>0$ be fixed. There exists $i$ such that $t_{i}^{\prime}, t_{i}^{\prime \prime} \in(t-\varepsilon, t)$. Now, by Lemma 5 , the set of all zeros of $X$ on $[0,+\infty)$ is zero-measured, due to the choice of sgn0. Hence, there exists $t^{\prime \prime} \in(t, t+\varepsilon)$ such that $X\left(t^{\prime \prime}\right) \neq 0$. One has $X\left(t_{i}^{\prime}\right) X\left(t^{\prime \prime}\right)<0$ or $X\left(t_{i}^{\prime \prime}\right) X\left(t^{\prime \prime}\right)<0$, which proves that $t \in Z$ and completes the proof.

The following result is proved in Shustin [17] too.

Lemma 7. If $t \in \operatorname{Acc} Z, t \geq h$, then $t-h \in \operatorname{Acc} Z$.

Proof. Indeed, due to Lemma 5 , if $t \in A c c Z$, then there exists for instance an increasing sequence $t_{i} \in Z$ with $X$ increasing around the points $t_{2 i}$ and decreasing around the points $t_{2 i+1}$ and such that $t_{i} \rightarrow t$ when $i \rightarrow+\infty$. Then, $\operatorname{sgn} X\left(t_{i}-h\right)=(-1)^{i+1}$, and there exists $t_{i}^{\prime} \in\left(t_{i}, t_{i+1}\right)$ such that $t_{i}^{\prime}-h \in Z$. Hence, $t-h=\lim _{i \rightarrow+\infty} t_{i}^{\prime}-h \in \operatorname{Acc} Z$.

\subsection{Principle of the proof}

\subsubsection{Preliminary notations}

Let $t, b>0$ be such that $X(t)=X(t+b)=0$. When $X>0$ on $(t, t+b)$ and $X$ is increasing and then decreasing on $[t, t+b]$, one denotes $\mathcal{G}_{-}^{P}(b)$ (resp. $\left.\mathcal{G}_{+}^{P}(b)\right)$ the time length of the increasing (resp. decreasing) phase. When $X<0$ on $(t, t+b)$ and $X$ is decreasing and then increasing, one denotes $\mathcal{G}_{-}^{N}(b)$ (resp. $\mathcal{G}_{+}^{N}(b)$ ) the time length of the decreasing (resp. increasing) phase. Simple computations lead to:

$$
\mathcal{G}_{ \pm}^{P} \triangleq \mathcal{F}_{\mp} \circ\left(\mathcal{F}_{+}+\mathcal{F}_{-}\right)^{-1}, \quad \mathcal{G}_{ \pm}^{N}(b) \triangleq-\mathcal{G}_{\mp}^{P}(-b)
$$


where $\mathcal{F}_{ \pm}$are defined in $(7)\left(P, N\right.$ stand for positive, negative). Remark that $\mathcal{G}_{ \pm}^{P}$ (resp. $\left.\mathcal{G}_{ \pm}^{N}\right)$ depend only upon $\left.F\right|_{\mathbb{R}^{+}}\left(\right.$resp. $\left.\left.F\right|_{\mathbb{R}^{-}}\right)$. When $F$ is odd, then $\mathcal{G}_{ \pm}^{P}=\mathcal{G}_{ \pm}^{N}$.

Below are listed some useful properties, whose proof is straightforward ( $\lambda$ is defined in (12)).

$$
\begin{gathered}
\left(\mathcal{G}_{ \pm}^{P}\right)^{\prime}=\frac{1 \pm F}{2} \circ\left(\mathcal{F}_{+}+\mathcal{F}_{-}\right)^{-1} \\
0<\lambda \leq\left(\mathcal{G}_{ \pm}^{a}\right)^{\prime} \leq 1-\lambda<1, \quad a \in\{P, N\} \\
\mathcal{G}_{-}^{a}+\mathcal{G}_{+}^{a}=\operatorname{Id}, \quad a \in\{P, N\}
\end{gathered}
$$

\subsubsection{Measures on the set of open intervals}

For any $\mathcal{C}^{1}$ convex function $\varphi$ on $\mathbb{R}^{+}$such that $\varphi(0)=\varphi^{\prime}(0)=0$, one denotes

$$
\varphi^{a}(b) \triangleq \int_{0}^{b} \varphi^{\prime} \circ \mathcal{G}_{-}^{a}\left(b^{\prime}\right) d b^{\prime}, \quad a \in\{P, N\}, b \in \mathbb{R}^{+} .
$$

One proves easily that for $a \in\{P, N\}, \varphi^{a}$ is convex, $\varphi^{a}(0)=\left(\varphi^{a}\right)^{\prime}(0)=0$, and

$$
\frac{1}{\lambda} \varphi(\lambda b) \leq \varphi^{a}(b) \leq \varphi(b) \quad \forall b \geq 0 .
$$

Definition 8. Let $\mu_{\varphi}$ denote the (unique) function on the set of bounded open intervals $I \subset[0,+\infty)$, such that

$$
\begin{aligned}
& \mu_{\varphi}(I)=\varphi^{P}(\text { meas } I) \quad \text { if } X>0 \text { a.e. on } I, \\
& \mu_{\varphi}(I)=\varphi^{N}(\text { meas } I) \quad \text { if } X<0 \text { a.e. on } I, \\
& \mu_{\varphi}(I)=\sum_{\begin{array}{c}
J \text { connected } \\
\text { component of } I \backslash Z
\end{array}} \mu_{\varphi}(J) \quad \text { for all } I .
\end{aligned}
$$

For any bounded open interval I, one denotes

$$
\begin{aligned}
& \mu_{p}(I) \triangleq \mu_{\left\{s \mapsto s^{p}\right\}}(I), p>1, \\
& \|I\|_{p} \triangleq\left(\sum_{\substack{J \text { connected } \\
\text { component of } I \backslash Z}}(\operatorname{meas} J)^{p}\right)^{\frac{1}{p}}, p \in[1,+\infty), \\
& \|I\|_{\infty} \triangleq \sup _{\begin{array}{c}
J \text { connected } \\
\text { component of } I \backslash Z
\end{array}} \text { meas } J .
\end{aligned}
$$

RR $n^{\circ} 3443$ 
For any open interval $I$, the set $I \backslash Z$ is open, since $Z$ is closed. Hence, it has at most a countable number of connected components $J$, which are open intervals. Since $\mu_{\varphi}(J) \geq 0$ and meas $J \geq 0$, the sums on $J$ in Definition 8 do not depend upon the ordering of the connected components. Hence, $\mu_{\varphi}(I)$ and $\|I\|_{p}$ are well-defined. Moreover, if $I$ is bounded, then meas $I<+\infty,\|I\|_{p} \leq\|I\|_{1}=$ meas $I<+\infty$ (as meas $Z=0$ by Lemma 5 ), and $\mu_{\varphi}(I) \leq \sum_{J} \varphi($ meas $J) \leq \varphi($ meas $I)<+\infty$ (by the convexity of $\varphi$ and $\varphi(0)=0$ ).

Note that $\left(\mu_{p}(I)\right)^{\frac{1}{p}}$ and $\|I\|_{p}$ are "equivalent":

$$
\lambda^{1-\frac{1}{p}}\|I\|_{p} \leq\left(\mu_{p}(I)\right)^{\frac{1}{p}} \leq\|I\|_{p} .
$$

In the sequel, we shall denote also $\|\cdot\|_{p}$ the usual $l^{p}$-norm of sequences, when no ambiguity is possible.

\subsubsection{The central intermediate result}

In order to bound Acc $Z$, let us fix $\alpha \in \operatorname{Acc} Z, \alpha \geq 2 h$. By Lemma 7, $\alpha-k h \in$ Acc $Z$ for all $k \in \mathbb{N}, k \leq \frac{\alpha}{h}$. In the following, one exhibits a function $\mu$ of $\alpha \in \operatorname{Acc} Z \cap[h,+\infty)$ (depending on $Z$ ), such that on the one hand $\mu(\alpha-k h)$ decreases and "tends to 0 when $k \rightarrow+\infty$ ", and on the other hand, $\mu\left(\alpha-k_{0} h\right)$ is lower bounded by some constant (depending on $\delta$ ), for $k_{0}=\left\lfloor\frac{\alpha}{h}\right\rfloor-1$. Here, $\left\lfloor\frac{\alpha}{h}\right\rfloor$ denotes the integer part of $\frac{\alpha}{h}$. This furnishes a bound for $k_{0}$ and then for $\alpha$.

Proposition 9. Let $\varphi$ be as before. Let $\gamma \in(0,1], p \in(1,+\infty]$. Then, there exists $\theta>0$ depending on $\gamma, p$ and $\sup _{x \in \mathbb{R}}|F(x)|$ only, such that, for any open interval $I \subset[h,+\infty)$ with bounds in Acc $Z$ and length meas $I \leq h$,

$$
\begin{gathered}
\mu_{\varphi}(I-h) \leq \mu_{\varphi}(I) \\
\mu_{\gamma+1}(I-h) \leq \mu_{\gamma+1}(I)-\theta \frac{\|I\|_{p}^{\frac{3}{p-1}+2+\gamma}}{(\text { meas } I)^{\frac{3}{p-1}+1}} .
\end{gathered}
$$

In Proposition 9 and in the sequel, one denotes $A \pm t=\left\{t^{\prime} \pm t: t^{\prime} \in A\right\}$ for any $t \in \mathbb{R}$ and $A$ subset of $\mathbb{R}$.

Applying (17) to

$$
I_{k} \triangleq(\alpha-(k+1) h, \alpha-k h), \quad k=0, \ldots, k_{0}-1,
$$

one gets

$$
\mu_{\gamma+1}\left(I_{k_{0}}\right) \leq \mu_{\gamma+1}\left(I_{0}\right)-\frac{\theta}{h^{\frac{3}{p-1}+1}} \sum_{k=0}^{k_{0}-1}\left\|I_{k}\right\|_{p}^{\frac{3}{p-1}+2+\gamma}
$$


Using formulas (15) and (16) for $p \in(1,+\infty)$, one obtains

$$
\begin{aligned}
\sum_{k=0}^{k_{0}-1}\left\|I_{k}\right\|_{p}^{\frac{3}{p-1}+2+\gamma} & \geq \sum_{k=0}^{k_{0}-1}\left(\mu_{p}\left(I_{k}\right)\right)^{\frac{1}{p}\left(\frac{3}{p-1}+2+\gamma\right)} \\
& \geq k_{0}\left(\mu_{p}\left(I_{k_{0}}\right)\right)^{\frac{1}{p}\left(\frac{3}{p-1}+2+\gamma\right)} \\
& \geq k_{0} \lambda^{\frac{1-\gamma}{p}+2+\gamma}\left\|I_{k_{0}}\right\|_{p}^{\frac{3}{p-1}+2+\gamma} .
\end{aligned}
$$

Passing to the limit, the resulting inequality is also valid for $p=+\infty$. Since $\mu_{\gamma+1}\left(I_{0}\right) \leq$ $\left\|I_{0}\right\|_{\gamma+1}^{\gamma+1} \leq\left\|I_{0}\right\|_{1}^{\gamma+1}=h^{\gamma+1}$, one gets

$$
k_{0} \leq \frac{1}{\theta \lambda^{\frac{1-\gamma}{p}+2+\gamma}}\left(\frac{h}{\left\|I_{k_{0}}\right\|_{p}}\right)^{\frac{3}{p-1}+2+\gamma} .
$$

Let us bound $\left\|I_{k_{0}}\right\|_{p}$ from below wrt $\delta$. The connected components of $Z^{\mathrm{c}}=\mathbb{R}^{+} \backslash Z$ are the maximal intervals where $X$ has a constant sign a.e. Let $I$ be a bounded interval of $(-h,+\infty)$ with e.g. $\operatorname{sgn} X(t)=1$ a.e. on $I$. Then, $X$ decreases in $I+h$, which implies that either $X$ has constant sign on $I+h$, or $X$ is positive on the first part of the interval $I+h$ and negative on the other part. Hence, $I+h$ is included in the union of the closure of two connected components of $Z^{\mathrm{c}}$. This implies that $\delta$ verifies:

$$
\begin{aligned}
\delta & =\sup \{\text { meas } I: I \text { interval } \subset(-h, 0), \operatorname{sgn} X \text { constant a.e. on } I\} \\
& \leq 2 \sup \{\text { meas } I: I \text { connected component of }(0, h) \backslash Z\} \\
& \leq 4 \sup \left\{\text { meas } I: I \text { connected component of }\left(\alpha-\left(k_{0}+1\right) h, \alpha-k_{0} h\right) \backslash Z\right\} \\
& =4\left\|I_{k_{0}}\right\|_{\infty} \leq 4\left\|I_{k_{0}}\right\|_{p} .
\end{aligned}
$$

The second inequality is deduced from the fact that $\left(\alpha-\left(k_{0}+1\right) h, \alpha-k_{0} h\right) \subset(0,2 h)$. Therefore,

$$
k_{0} \leq \frac{1}{\theta \lambda^{\frac{1-\gamma}{p}+2+\gamma}}\left(\frac{4 h}{\delta}\right)^{\frac{3}{p-1}+2+\gamma}
$$

so $\max \operatorname{Acc} Z \leq\left(k_{0}+2\right) h \leq h C_{p, \gamma}\left(1+(h / \delta)^{\frac{3}{p-1}+2+\gamma}\right)$ for a certain constant $C_{p, \gamma}>0$ depending on $p, \gamma$ and $\lambda$ only. The proof of Theorem 2 is achieved taking $p=+\infty$ and $\gamma=\varepsilon$.

\subsubsection{Overview of the proof of Proposition 9}

In order to end the proof of Theorem 2, it only remains to prove Proposition 9. This is done in four steps, in Sections 4.3 to 4.6. Let us give an overview of this proof.

When $I$ and $(I-h)$ do not contain accumulation points of $Z$, their connected components may be ordered, together with the sums defining the measures involved in (16) and (17). For 
the particular case where $X$ changes only one time its sense of variation on any subinterval of $I$ where its sign is constant (that is when any connected component of $I \backslash Z$ intersects no more than two connected components of $I \backslash(Z+h)$ ), the proof is treated in Section 4.3.

When some connected components of $I \backslash Z$ intersect more than two connected components of $I \backslash(Z+h)$, but always a finite number of them, which is the case when $I-h$ does not contain accumulation points of $Z$, the computations are more cumbersome. They are presented in Section 4.4. Note that this part does not use Section 4.3: the particular case presented therein is simpler, and one provides it for pedagogical purpose rather.

The next step (in Section 4.5) consists in allowing an infinite number of changes of variation on subintervals of $I$ where $X$ has a constant sign, that is to eliminate the restrictions on $I-h$. The proof in this case follows from the results of Section 4.4, by an approximation process.

Last, the case where $I$ contains accumulation points of $Z$ is treated in Section 4.6: a summation of contributions coming from each connected component of $I \backslash$ Acc $Z$, that are treated by the results of Section 4.5, is achieved. This ends the proof of Proposition 9.

Independently, one presents in Section 4.7 a simpler variant of Sections 4.4 and 4.5, in the case where $F$ is odd. It consists in proving that, given the set $I \backslash Z$, the value of $\mu_{\varphi}(I-h)$ obtained in the particular case of Section 4.3 is indeed the maximal that may be obtained. The technique uses Schur convexity as a central tool.

\subsection{Proof of Proposition 9 in a particular case where $I$ and $I-h$ do not contain accumulation points of $Z$}

If $I$ is an open interval with bounds in $\operatorname{Acc} Z$ such that $I \cap \operatorname{Acc} Z=\emptyset$, then the points of $Z \cap I$ may be ordered in increasing order. Therefore, the connected components of the open set $U \triangleq I \backslash Z$ may be ordered in increasing order too. Let us denote them by $U_{j}, j \in \mathcal{J} \subset \mathbb{Z}$ ( $U_{j}$ increasing wrt $j$ ), and put $U_{j}=\emptyset$ for $j \in \mathbb{Z} \backslash \mathcal{J}$. Since, by the definition of $Z$, the sign of $X$ is constant a.e. in $U_{j}$ and alternating wrt $j$, one imposes e.g. $\operatorname{sgn} X=(-1)^{j}$ a.e. on $U_{j}$.

Let us define the sequences $a$ and $b$ by: $a_{j} \triangleq P$ if $j$ is even, $a_{j} \triangleq N$ if $j$ is odd, $j \in \mathbb{Z}$, and $b_{j} \triangleq$ meas $U_{j}$ for $j \in \mathbb{Z}$. One has meas $I=\|b\|_{1},\|I\|_{p}=\|b\|_{p}$, and $\mu_{\varphi}(I)=\mu_{\varphi}(b) \triangleq$ $\sum_{j \in \mathbb{Z}} \varphi^{a_{j}}\left(b_{j}\right)$.

Suppose now that $I-h$ does not contain accumulation points of $Z$ too. Denote $\tilde{U}_{j}$, $j \in \tilde{\mathcal{J}} \subset \mathbb{Z}$, the connected components of $\tilde{U} \triangleq I \backslash(Z+h)$, increasingly ordered as the $U_{j}$, and put $\tilde{U}_{j}=\emptyset$ for $j \in \mathbb{Z} \backslash \tilde{\mathcal{J}}$. Let $\tilde{b}$ be the sequence defined by: $\tilde{b}_{j} \triangleq$ meas $\tilde{U}_{j}$ for $j \in \mathbb{Z}$. One has $\mu_{\varphi}(I-h)=\mu_{\varphi}(\tilde{b})=\sum_{j \in \mathbb{Z}} \varphi^{a_{j}}\left(\tilde{b}_{j}\right)$. Since $X$ increases (resp. decreases) around $t \in I$ if and only if $X(.-h)<0$ (resp. $X(.-h)>0$ ) a.e. around $t$, the sets $\tilde{U}_{j}, j \in \tilde{\mathcal{J}}$, are exactly the connected components of the open subset of $I$ containing all the points around which $X$ is monotonous. The sequence $\tilde{b}$ verifies the following property: there exists $\tilde{X} \in W^{1, \infty}(I)$ 
such that, for all $j \in \mathbb{Z}$,

$$
\dot{\tilde{X}}=(-1)^{j-1}+F(\tilde{X}) \text { a.e. on } \tilde{U}_{j},(-1)^{j} \tilde{X}>0 \text { a.e. on } U_{j} .
$$

Any sequence verifying this property is called a predecessor of $b$. A particularly simple predecessor, denoted $b^{\prime}$, is obtained by using a function $\tilde{X}$ satisfying (18) and being either increasing/decreasing, or decreasing/increasing, on any interval $U_{j}$. Let us denote $X^{\prime}$ the corresponding value of $\tilde{X}$. One shows easily (see Figure 1) that this corresponds to choose

$$
b_{2 j-1}^{\prime}=\mathcal{G}_{-}^{P}\left(b_{2 j}\right)+\mathcal{G}_{+}^{N}\left(b_{2 j-1}\right), \quad b_{2 j}^{\prime}=\mathcal{G}_{-}^{N}\left(b_{2 j+1}\right)+\mathcal{G}_{+}^{P}\left(b_{2 j}\right),
$$

that is

$$
b_{j}^{\prime}=\mathcal{G}_{-}^{a_{j+1}}\left(b_{j+1}\right)+\mathcal{G}_{+}^{a_{j}}\left(b_{j}\right)=b_{j}+\mathcal{G}_{-}^{a_{j+1}}\left(b_{j+1}\right)-\mathcal{G}_{-}^{a_{j}}\left(b_{j}\right)
$$

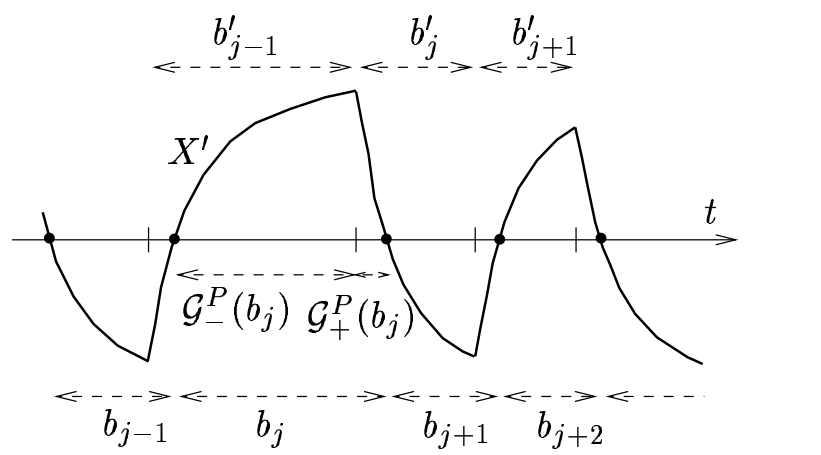

Figure 1: The predecessor $b^{\prime}$ (the indexation shown corresponds to an even integer $j$ )

In this section, one proves Proposition 9 in the particular case where $\tilde{b}=b^{\prime}$.

Using the identity

$$
\varphi^{a}\left(s_{1}+s_{2}-s_{3}\right)=\varphi^{a}\left(s_{1}\right)+[\varphi(s)]_{s_{3}}^{s_{2}}+\int_{s_{3}}^{s_{2}}\left(\varphi^{\prime} \circ \mathcal{G}_{-}^{a}\left(s+s_{1}-s_{3}\right)-\varphi^{\prime}(s)\right) d s,
$$

one gets

$$
\varphi^{a_{j}}\left(b_{j}^{\prime}\right)=\varphi^{a_{j}}\left(b_{j}\right)+[\varphi(s)]_{\mathcal{G}_{-}^{a_{j}}\left(b_{j}\right)}^{\mathcal{G}_{a_{j+1}}\left(b_{j+1}\right)}+\int_{\mathcal{G}_{-}^{a_{j}}\left(b_{j}\right)}^{\mathcal{G}_{-}^{a_{j+1}}\left(b_{j+1}\right)}\left(\varphi^{\prime} \circ \mathcal{G}_{-}^{a_{j}}\left(s+\mathcal{G}_{+}^{a_{j}}\left(b_{j}\right)\right)-\varphi^{\prime}(s)\right) d s
$$

$\mathrm{RR} \quad \mathrm{n}^{\circ} 3443$ 
Let us study the last term of (21). If e.g. $\mathcal{G}_{-}^{a_{j}}\left(b_{j}\right) \leq \mathcal{G}_{-}^{a_{j+1}}\left(b_{j+1}\right)$, then one has, for any $s \in\left[\mathcal{G}_{-}^{a_{j}}\left(b_{j}\right), \mathcal{G}_{-}^{a_{j+1}}\left(b_{j+1}\right)\right]$,

$\mathcal{G}_{-}^{a_{j}}\left(b_{j}\right) \leq s \Rightarrow \mathcal{G}_{+}^{a_{j}}\left(b_{j}\right) \leq \mathcal{G}_{+}^{a_{j}} \circ\left(\mathcal{G}_{-}^{a_{j}}\right)^{-1}(s) \Rightarrow s+\mathcal{G}_{+}^{a_{j}}\left(b_{j}\right) \leq\left(\mathcal{G}_{-}^{a_{j}}\right)^{-1}(s) \Rightarrow \mathcal{G}_{-}^{a_{j}}\left(s+\mathcal{G}_{+}^{a_{j}}\left(b_{j}\right)\right) \leq s$,

using $\mathcal{G}_{-}^{a}+\mathcal{G}_{+}^{a}=\operatorname{Id}$ and the fact that the functions $\mathcal{G}_{ \pm}^{a}$ are increasing. More precisely, using the bounds on the derivatives, one gets:

$$
\begin{aligned}
\mathcal{G}_{-}^{a_{j}}\left(s+\mathcal{G}_{+}^{a_{j}}\left(b_{j}\right)\right)-s & =\mathcal{G}_{-}^{a_{j}}\left(s+\mathcal{G}_{+}^{a_{j}}\left(b_{j}\right)\right)-\mathcal{G}_{-}^{a_{j}}\left(s+\mathcal{G}_{+}^{a_{j}} \circ\left(\mathcal{G}_{-}^{a_{j}}\right)^{-1}(s)\right) \\
& \leq \frac{\lambda^{2}}{(1-\lambda)}\left(\mathcal{G}_{-}^{a_{j}}\left(b_{j}\right)-s\right) \leq 0 .
\end{aligned}
$$

Then, by the convexity of $\varphi$, one obtains

$$
\varphi^{\prime} \circ \mathcal{G}_{-}^{a_{j}}\left(s+\mathcal{G}_{+}^{a_{j}}\left(b_{j}\right)\right)-\varphi^{\prime}(s) \leq 0,
$$

and the last term of (21) is nonpositive. A similar proof holds when $\mathcal{G}_{-}^{a_{j}}\left(b_{j}\right) \geq \mathcal{G}_{-}^{a_{j+1}}\left(b_{j+1}\right)$. Hence, one obtains by addition of $(21): \mu_{\varphi}\left(b^{\prime}\right) \leq \mu_{\varphi}(b)$, that is (16), using the fact that $\varphi\left(\mathcal{G}_{-}^{a_{j}}\left(b_{j}\right)\right) \rightarrow 0$ when $|j| \rightarrow+\infty$.

To prove (17), consider the sequence $c_{j} \triangleq \mathcal{G}_{-}^{a_{j}}\left(b_{j}\right)$. The previous computations lead to

$$
\mu_{\varphi}\left(b^{\prime}\right) \leq \mu_{\varphi}(b)+\sum_{j \in \mathbb{Z}} \int_{c_{j}}^{c_{j+1}}\left(\varphi^{\prime}\left(s+\frac{\lambda^{2}}{(1-\lambda)}\left(c_{j}-s\right)\right)-\varphi^{\prime}(s)\right) d s .
$$

For $\varphi(s)=s^{\gamma+1}$, bounding $\varphi^{\prime \prime}$ from below, one deduces:

$$
\mu_{\varphi}\left(b^{\prime}\right) \leq \mu_{\varphi}(b)-\gamma(\gamma+1)\left(\sup _{j \in \mathbb{Z}} c_{j}\right)^{\gamma-1} \frac{\lambda^{2}}{(1-\lambda)} \sum_{j \in \mathbb{Z}} \frac{\left(c_{j}-c_{j+1}\right)^{2}}{2} .
$$

Let us now use (with $q=2$ ) the following interpolation inequality, which is a discrete analog of a result by Gagliardo [8] and Nirenberg [13]. A proof is given in Appendix.

Lemma 10. Let $1<p \leq+\infty$ and $1<q<+\infty$. Then, there exists $C_{p, q}>0$ such that, for any nonzero real sequence $\left(b_{j}\right)_{j \in \mathbb{Z}}$, the following formula is true, as soon as the involved norms are finite:

$$
\left\|b_{j}-b_{j-1}\right\|_{q}^{q} \geq C_{p, q} \frac{\|b\|_{p^{\frac{p(2 q-1)}{p-1}}}}{\|b\|_{1}^{\frac{p q-p+q}{p-1}}}
$$

One gets

$$
\sum_{j \in \mathbb{Z}}\left(c_{j}-c_{j+1}\right)^{2} \geq C_{p, 2} \frac{\|c\|_{p}^{\frac{3 p}{p-1}}}{\|c\|_{1}^{\frac{p+2}{p-1}}}
$$


As $\left(\sup c_{j}\right)^{\gamma-1} \geq\|c\|_{p}^{\gamma-1}$, one deduces

$$
\mu_{\gamma+1}\left(b^{\prime}\right) \leq \mu_{\gamma+1}(b)-\theta^{\prime} \frac{\|c\|_{p}^{\frac{3}{p-1}+2+\gamma}}{\|c\|_{1}^{\frac{3}{p-1}}+1},
$$

where $\theta^{\prime}>0$ depends on $\gamma, p$ and $\lambda$. Using the inequalities on $\left(\mathcal{G}_{-}^{a}\right)^{\prime}$ and the fact that $\mathcal{G}_{-}^{a}(0)=0$, one gets

$$
\|c\|_{p} \geq \lambda\|b\|_{p}, \quad\|c\|_{1} \leq(1-\lambda)\|b\|_{1}
$$

which gives (17) in the case where $\tilde{b}=b^{\prime}$.

\subsection{Proof of Proposition 9 for general $I$ such that $I$ and $I-h$ do not contain accumulation points of $Z$}

One studies here the case where $I \cap \operatorname{Acc} Z=I \cap(\operatorname{Acc} Z+h)=\emptyset$, so the connected components of $U=I \backslash Z$ and $\tilde{U}=I \backslash(Z+h)$ may be ordered as in Section 4.3, but one supposes that the predecessor $\tilde{b}$ of $b$ is different (up to a translation) from $b^{\prime}$ defined in (19).

Let $U_{j}, b_{j}, \tilde{U}_{j}, \tilde{b}_{j}, a_{j}$ be defined as in Section 4.3. The function $\tilde{X}=\left.X\right|_{I}$ satisfies (18). Let us denote $t_{j}$ the right boundary of the interval $\tilde{U}_{j}$ (the $t_{j}$ are exactly the local extrema of $\tilde{X}$ ). Let us define also

$$
d_{j} \triangleq\left|\left(\mathcal{F}_{+}+\mathcal{F}_{-}\right)\left(\tilde{X}\left(t_{j}\right)\right)\right|
$$

The quantity $d_{j}$ is the time necessary for a solution of (1)-(5) to go from 0 to $\tilde{X}\left(t_{j}\right)$ and back to 0 with only one change of the sense of variation. For any $j \in \mathbb{Z}$, let us denote $\tilde{\jmath}=\tilde{\jmath}(j)$ the unique index such that $\tilde{U}_{\tilde{\jmath}} \cap U_{j} \neq \emptyset$ and $\tilde{U}_{\tilde{\jmath}} \cap U_{j+1} \neq \emptyset$. The parity of $j$ and $\tilde{\jmath}$ is the same. The maximal integer $l$ such that $\tilde{U}_{\tilde{\jmath}-l} \subset U_{j}$ is even and denoted $2 \bar{l}=2 \bar{l}(j)$. One has $\tilde{U}_{\tilde{\jmath}-l} \subset U_{j}$ for $l=1, \ldots, 2 \bar{l}$, and $\tilde{\jmath}(j)-2 \bar{l}(j)-1=\tilde{\jmath}(j-1)$. For all the notations, see Figure 2.

To compare, as in Section $4.3, \mu_{\varphi}(\tilde{b})$ and $\mu_{\varphi}(b)$, one remarks that

$$
\sum_{j \in \mathbb{Z}} \varphi^{a_{j}}\left(\tilde{b}_{j}\right)=\sum_{j \in \mathbb{Z}} \sum_{l=0}^{2 \bar{l}(j)} \varphi^{a_{j-l}}\left(\tilde{b}_{\tilde{\jmath}(j)-l}\right) .
$$

Then, one is led to compare $\sum_{l=0}^{2 \bar{l}(j)} \varphi^{a_{j-l}}\left(\tilde{b}_{\tilde{\jmath}(j)-l}\right)$ and $\varphi^{a_{j}}\left(b_{j}\right)$. One has (see Figure 2)

$$
\begin{aligned}
\tilde{b}_{\tilde{\jmath}} & =\mathcal{G}_{-}^{a_{j+1}}\left(d_{\tilde{\jmath}}\right)+\mathcal{G}_{+}^{a_{j}}\left(d_{\tilde{\jmath}-1}\right)=d_{\tilde{\jmath}-1}+\mathcal{G}_{-}^{a_{j+1}}\left(d_{\tilde{\jmath}}\right)-\mathcal{G}_{-}^{a_{j}}\left(d_{\tilde{\jmath}-1}\right) \\
\tilde{b}_{\tilde{\jmath}-l} & =\mathcal{G}_{-}^{a_{j}}\left(d_{\tilde{\jmath}-l}\right)-\mathcal{G}_{-}^{a_{j}}\left(d_{\tilde{\jmath}-l-1}\right) \quad \text { for } l \text { odd in }\{1, \ldots, 2 \bar{l}-1\} \\
\tilde{b}_{\tilde{\jmath}-l} & =-\mathcal{G}_{+}^{a_{j}}\left(d_{\tilde{\jmath}-l}\right)+\mathcal{G}_{+}^{a_{j}}\left(d_{\tilde{\jmath}-l-1}\right) \\
& =d_{\tilde{\jmath}-l-1}-d_{\tilde{\jmath}-l}+\mathcal{G}_{-}^{a_{j}}\left(d_{\tilde{\jmath}-l}\right)-\mathcal{G}_{-}^{a_{j}}\left(d_{\tilde{\jmath}-l-1}\right) \quad \text { for } l \text { even in }\{2, \ldots, 2 \bar{l}\} .
\end{aligned}
$$




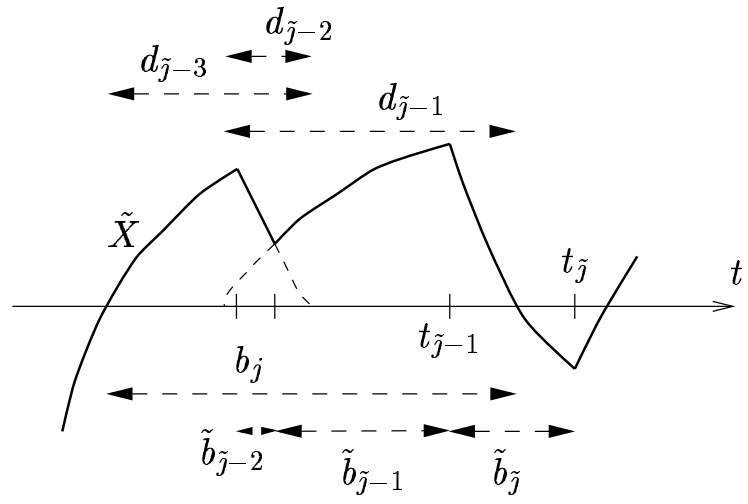

Figure 2: A predecessor $\tilde{b}$ (here $j$ is even, $\bar{l}(j)=1$ )

\subsubsection{Rewriting of $\mu_{\varphi}(\tilde{b})$ using identity (20)}

First, one has, for $l=0$ :

$$
\varphi^{a_{j}}\left(\tilde{b}_{\tilde{\jmath}}\right)=\varphi^{a_{j}}\left(d_{\tilde{\jmath}-1}\right)+[\varphi(s)]_{\mathcal{G}_{-}^{a_{j}}\left(d_{\tilde{\jmath}-1}\right)}^{\mathcal{G}_{a_{j+1}}\left(d_{\tilde{\jmath}}\right)}+r_{\tilde{\jmath}}
$$

where the rest $r_{\tilde{j}}$ is given by:

$$
r_{\tilde{\jmath}} \triangleq \int_{\mathcal{G}_{-}^{a_{j}}\left(d_{\tilde{\jmath}-1}\right)}^{\mathcal{G}_{-}^{a_{j+1}}\left(d_{\tilde{\jmath}}\right)}\left(\varphi^{\prime} \circ \mathcal{G}_{-}^{a_{j}}\left(s+\mathcal{G}_{+}^{a_{j}}\left(d_{\tilde{\jmath}-1}\right)\right)-\varphi^{\prime}(s)\right) d s .
$$

Second, for odd index $l$, one gets:

$$
\varphi^{a_{j-l}}\left(\tilde{b}_{\tilde{\jmath}-l}\right)=[\varphi(s)]_{\mathcal{G}_{-}^{a_{j}}\left(d_{\tilde{j}-l-1}\right)}^{\mathcal{G}_{j}}+r_{\tilde{\jmath}-l},
$$

where, for $l$ odd

$$
r_{\tilde{\jmath}-l} \triangleq \int_{\mathcal{G}_{-}^{a_{j}}\left(d_{\tilde{\jmath}-l-1}\right)}^{\mathcal{G}_{-}^{a_{j}}\left(d_{\tilde{\jmath}-l}\right)}\left(\varphi^{\prime} \circ \mathcal{G}_{-}^{a_{j+1}}\left(s-\mathcal{G}_{-}^{a_{j}}\left(d_{\tilde{\jmath}-l-1}\right)\right)-\varphi^{\prime}(s)\right) d s .
$$

Third, for $l$ nonzero and even, one has

$$
\begin{aligned}
\varphi^{a_{j-l}}\left(\tilde{b}_{\tilde{\jmath}-l}\right)=\varphi^{a_{j}}\left(d_{\tilde{\jmath}-l-1}\right. & \left.-d_{\tilde{\jmath}-l}\right)+[\varphi(s)]_{\mathcal{G}_{-}^{a_{j}}\left(d_{\tilde{\jmath}-l-1}\right)}^{\mathcal{G}_{j}}\left(d_{\tilde{\jmath}-l}\right) \\
& +\int_{\mathcal{G}_{-}^{a_{j}}\left(d_{\tilde{\jmath}-l-1}\right)}^{\mathcal{G}_{-}^{a_{j}}\left(d_{\tilde{\jmath}-l}\right)}\left(\varphi^{\prime} \circ \mathcal{G}_{-}^{a_{j}}\left(s+\mathcal{G}_{+}^{a_{j}}\left(d_{\tilde{\jmath}-l-1}\right)-d_{\tilde{\jmath}-l}\right)-\varphi^{\prime}(s)\right) d s .
\end{aligned}
$$

INRIA 
Adding the null term

$$
\begin{aligned}
\varphi^{a_{j}}\left(\sum_{l^{\prime}=0}^{l}(-1)^{l^{\prime}} d_{\tilde{\jmath}-l^{\prime}-1}\right)-\varphi^{a_{j}}\left(\sum_{l^{\prime}=0}^{l-2}(-1)^{l^{\prime}} d_{\tilde{\jmath}-l^{\prime}-1}\right) \\
\quad-\int_{d_{\tilde{\jmath}-l}}^{d_{\tilde{j}-l-1}} \varphi^{\prime} \circ \mathcal{G}_{-}^{a_{j}}\left(\sum_{l^{\prime}=0}^{l-1}(-1)^{l^{\prime}} d_{\tilde{\jmath}-l^{\prime}-1}+s\right) d s
\end{aligned}
$$

one gets:

$$
\varphi^{a_{j}-l}\left(\tilde{b}_{\tilde{\jmath}-l}\right)=\varphi^{a_{j}}\left(\sum_{l^{\prime}=0}^{l}(-1)^{l^{\prime}} d_{\tilde{\jmath}-l^{\prime}-1}\right)-\varphi^{a_{j}}\left(\sum_{l^{\prime}=0}^{l-2}(-1)^{l^{\prime}} d_{\tilde{\jmath}-l^{\prime}-1}\right)+[\varphi(s)]_{\mathcal{G}_{-}^{a_{j}}\left(d_{\tilde{\jmath}-l-1}\right)}^{\mathcal{G}_{j}^{a_{j}}\left(d_{\tilde{j}-l}\right)}+r_{\tilde{\jmath}-l},
$$

where, for $l$ nonzero and even

$$
\begin{aligned}
& r_{\tilde{\jmath}-l} \triangleq \int_{\mathcal{G}_{-}^{a_{j}}\left(d_{\tilde{\jmath}-l-1}\right)}^{\mathcal{G}_{j}^{a_{j}}\left(d_{\tilde{j}-l}\right)}\left(\varphi^{\prime} \circ \mathcal{G}_{-}^{a_{j}}\left(s+\mathcal{G}_{+}^{a_{j}}\left(d_{\tilde{\jmath}-l-1}\right)-d_{\tilde{\jmath}-l}\right)-\varphi^{\prime}(s)\right) d s \\
+ & \int_{d_{\tilde{\jmath}-l}}^{d_{\tilde{\jmath}-l-1}}\left(\varphi^{\prime} \circ \mathcal{G}_{-}^{a_{j}}\left(s-d_{\tilde{\jmath}-l}\right)-\varphi^{\prime} \circ \mathcal{G}_{-}^{a_{j}}\left(\sum_{l^{\prime}=0}^{l-1}(-1)^{l^{\prime}} d_{\tilde{\jmath}-l^{\prime}-1}+s\right)\right) d s=\int_{d_{\tilde{\jmath}-l}}^{d_{\tilde{\jmath}-l-1}} \psi_{j, l}(s) d s
\end{aligned}
$$

and

$$
\begin{aligned}
\psi_{j, l}(s) \triangleq\left(\mathcal{G}_{-}^{a_{j}}\right)^{\prime}(s)\left[\varphi^{\prime} \circ \mathcal{G}_{-}^{a_{j}}(s)\right. & \left.-\varphi^{\prime} \circ \mathcal{G}_{-}^{a_{j}}\left(\mathcal{G}_{-}^{a_{j}}(s)-d_{\tilde{\jmath}-l}+\mathcal{G}_{+}^{a_{j}}\left(d_{\tilde{\jmath}-l-1}\right)\right)\right] \\
& +\varphi^{\prime} \circ \mathcal{G}_{-}^{a_{j}}\left(s-d_{\tilde{\jmath}-l}\right)-\varphi^{\prime} \circ \mathcal{G}_{-}^{a_{j}}\left(\sum_{l^{\prime}=0}^{l-1}(-1)^{l^{\prime}} d_{\tilde{\jmath}-l^{\prime}-1}+s\right) .
\end{aligned}
$$

By summation over all the indexes $l$, one gets:

$$
\sum_{l=0}^{2 \bar{l}} \varphi^{a_{j-l}}\left(\tilde{b}_{\tilde{\jmath}-l}\right)=\varphi^{a_{j}}\left(\sum_{l=0}^{2 \bar{l}}(-1)^{l} d_{\tilde{\jmath}-l-1}\right)+[\varphi(s)]_{\mathcal{G}_{-}^{a_{j}}\left(d_{\tilde{\jmath}-2 \bar{l}-1}\right)}^{\mathcal{G}_{j+1}\left(d_{\tilde{\jmath}}\right)}+\sum_{l=0}^{2 \bar{l}} r_{\tilde{\jmath}-l}
$$

As (cf. Figure 2)

$$
\sum_{l=0}^{2 \bar{l}}(-1)^{l} d_{\tilde{\jmath}-l-1}=b_{j}
$$

and

$$
\tilde{\jmath}(j)-2 \bar{l}(j)-1=\tilde{\jmath}(j-1),
$$

one finally may write:

$$
\mu_{\varphi}(\tilde{b})=\sum_{j \in \mathbb{Z}} \sum_{l=0}^{2 \bar{l}(j)} \varphi^{a_{j-l}}\left(b_{\tilde{\jmath}(j)-l}\right)=\mu_{\varphi}(b)+\sum_{j \in \mathbb{Z}} \sum_{l=0}^{2 \bar{l}(j)} r_{\tilde{\jmath}(j)-l} .
$$

$\mathrm{RR} \mathrm{n}^{\circ} 3443$ 


\subsubsection{Bounding of the rest}

Let us consider the sequence $\left(c_{\frac{j}{3}}\right)_{j \in \mathbb{Z}}$, defined for $j \in \mathbb{Z}$ by:

$$
\begin{aligned}
c_{j} & \triangleq \mathcal{G}_{-}^{a_{j}}\left(b_{j}\right) \quad(\text { as in Section } 4.3), \\
c_{j+\frac{1}{3}} & \triangleq \mathcal{G}_{-}^{a_{j}}\left(d_{\tilde{\jmath}(j)-1}\right) \leq c_{j} \quad\left(\text { since } d_{\tilde{\jmath}(j)-1} \leq b_{j}\right), \\
c_{j-\frac{1}{3}} & \triangleq \mathcal{G}_{-}^{a_{j}}\left(d_{\tilde{\jmath}(j)-2 \bar{l}(j)-1}\right) \leq c_{j} \quad\left(\text { since } d_{\tilde{\jmath}(j)-2 \bar{l}(j)-1} \leq b_{j}\right) .
\end{aligned}
$$

Lemma 11. For any $l=0, \ldots, 2 \bar{l}, r_{\tilde{\jmath}-l} \leq 0$. Moreover, when $\varphi(s)=s^{\gamma+1}$, there exists $C_{\gamma, \lambda}>0$, such that for all $j \in \mathbb{Z}$,

$$
\begin{gathered}
r_{\tilde{\jmath}(j)} \leq-C_{\gamma, \lambda} \max \left\{c_{j}, c_{j+1}\right\}^{\gamma-1}\left(c_{j+\frac{2}{3}}-c_{j+\frac{1}{3}}\right)^{2}, \\
\sum_{l=1}^{\bar{l}(j)} r_{\tilde{\jmath}(j)-2 l} \leq-C_{\gamma, \lambda} c_{j}^{\gamma-1}\left(c_{j+\frac{1}{3}}-c_{j}\right)^{2}, \\
\sum_{l=1}^{2 \bar{l}(j)} r_{\tilde{\jmath}(j)-l} \leq-C_{\gamma, \lambda} c_{j}^{\gamma-1}\left(c_{j}-c_{j-\frac{1}{3}}\right)^{2} .
\end{gathered}
$$

From Lemma 11, all the $r_{\tilde{\jmath}-l}$ are nonpositive, then $\mu_{\varphi}(\tilde{b}) \leq \mu_{\varphi}(b)$ and $(16)$ is deduced.

Now, for $\varphi(s)=s^{\gamma+1}$, adding the three inequalities of Lemma 11, and then summing on $j \in \mathbb{Z}$, one gets

$$
2 \sum_{j \in \mathbb{Z}} \sum_{l=0}^{2 \bar{l}(j)} r_{\tilde{\jmath}(j)-l} \leq-C_{\gamma, \lambda}\left\|c_{j}\right\|_{\infty}^{\gamma-1} \sum_{j \in \frac{1}{3} \mathbb{Z}}\left(c_{j}-c_{j-\frac{1}{3}}\right)^{2} \leq-2 \theta^{\prime \prime}\left\|c_{j}\right\|_{\infty}^{\gamma-1} \frac{\left\|c_{\frac{j}{3}}\right\|_{p}^{\frac{3}{p-1}+3}}{\left\|c_{\frac{j}{3}}\right\|_{1}^{\frac{3}{p-1}+1}},
$$

by Lemma 10 , where $\theta^{\prime \prime}>0$ depends upon $\lambda, \gamma$ and $p$. Since $\left\|c_{\frac{j}{3}}\right\|_{p} \geq\left\|c_{j}\right\|_{p},\left\|c_{\frac{j}{3}}\right\|_{1} \leq 3\left\|c_{j}\right\|_{1}$ (as $c_{j} \geq c_{j+\frac{1}{3}}, c_{j-\frac{1}{3}}$ ), and $\sup _{j \in \mathbb{Z}} c_{j} \leq\left\|c_{j}\right\|_{p}$, one obtains:

$$
\mu_{\gamma+1}(\tilde{b}) \leq \mu_{\gamma+1}(b)-\frac{\theta^{\prime \prime}}{3^{\frac{3}{p-1}+1}} \frac{\left\|c_{j}\right\|_{p}^{\frac{3}{p-1}+2+\gamma}}{\left\|c_{j}\right\|_{1}^{\frac{3}{p-1}+1}}
$$

which is analogous to (22), and one ends the proof as in Section 4.3.

Proof of Lemma 11.

- When $l=0$, by the same proof as in Section 4.3 , one may show that $r_{\tilde{\jmath}} \leq 0$ for any $\left(\mathcal{C}^{1}\right.$ convex) $\varphi$ and that, for $\varphi(s)=s^{\gamma+1}$ :

$$
\begin{aligned}
r_{\tilde{\jmath}} & \leq-\gamma(\gamma+1) \frac{\lambda^{2}}{2(1-\lambda)} \max \left\{\mathcal{G}_{-}^{a_{j}}\left(d_{\tilde{\jmath}-1}\right), \mathcal{G}_{-}^{a_{j+1}}\left(d_{\tilde{\jmath}}\right)\right\}^{\gamma-1}\left(\mathcal{G}_{-}^{a_{j+1}}\left(d_{\tilde{\jmath}}\right)-\mathcal{G}_{-}^{a_{j}}\left(d_{\tilde{\jmath}-1}\right)\right)^{2} \\
& \leq-\gamma(\gamma+1) \frac{\lambda^{2}}{2(1-\lambda)} \max \left\{c_{j+\frac{1}{3}}, c_{j+\frac{2}{3}}\right\}^{\gamma-1}\left(c_{j+\frac{2}{3}}-c_{j+\frac{1}{3}}\right)^{2} .
\end{aligned}
$$


Formula (24) is then deduced from the inequalities: $c_{j+\frac{2}{3}} \leq c_{j+1}, c_{j+\frac{1}{3}} \leq c_{j}$.

- If $l$ is odd, then $d_{\tilde{\jmath}-l} \geq d_{\tilde{\jmath}-l-1}$ and, for $s \in\left[\mathcal{G}_{-}^{a_{j}}\left(d_{\tilde{\jmath}-l-1}\right), \mathcal{G}_{-}^{a_{j}}\left(d_{\tilde{\jmath}-l}\right)\right]$, one has

$$
0 \leq \mathcal{G}_{-}^{a_{j+1}}\left(s-\mathcal{G}_{-}^{a_{j}}\left(d_{\tilde{\jmath}-l-1}\right)\right) \leq(1-\lambda)\left(s-\mathcal{G}_{-}^{a_{j}}\left(d_{\tilde{\jmath}-l-1}\right)\right) \leq s .
$$

Hence, $r_{\tilde{\jmath}-l} \leq 0$ for any $\varphi$, and, when $\varphi(s)=s^{\gamma+1}$, using the previous inequalities, $\mathcal{G}_{-}^{a_{j}}(0)=0$ and $\lambda<1$, one gets:

$$
\begin{aligned}
r_{\tilde{\jmath}-l} \leq & -\gamma(\gamma+1)\left(\mathcal{G}_{-}^{a_{j}}\left(d_{\tilde{\jmath}-l}\right)\right)^{\gamma-1}\left[\frac{\lambda}{2}\left(\mathcal{G}_{-}^{a_{j}}\left(d_{\tilde{\jmath}-l}\right)-\mathcal{G}_{-}^{a_{j}}\left(d_{\tilde{\jmath}-l-1}\right)\right)^{2}\right. \\
& \left.+\mathcal{G}_{-}^{a_{j}}\left(d_{\tilde{\jmath}-l-1}\right)\left(\mathcal{G}_{-}^{a_{j}}\left(d_{\tilde{\jmath}-l}\right)-\mathcal{G}_{-}^{a_{j}}\left(d_{\tilde{\jmath}-l-1}\right)\right)\right] \\
\leq & -\gamma(\gamma+1)\left(\mathcal{G}_{-}^{a_{j}}\left(b_{j}\right)\right)^{\gamma-1} \lambda^{2}\left(\frac{\lambda}{2}\left(d_{\tilde{\jmath}-l}-d_{\tilde{\jmath}-l-1}\right)^{2}+d_{\tilde{\jmath}-l-1}\left(d_{\tilde{\jmath}-l}-d_{\tilde{\jmath}-l-1}\right)\right) \\
\leq & -\gamma(\gamma+1) \frac{\lambda^{3}}{2} c_{j}^{\gamma-1}\left(d_{\tilde{\jmath}-l}^{2}-d_{\tilde{\jmath}-l-1}^{2}\right) .
\end{aligned}
$$

- If $l$ is nonzero and even, then $d_{\tilde{\jmath}-l} \leq d_{\tilde{\jmath}-l-1}$. Let us rewrite

$$
\begin{aligned}
\psi_{j, l}(s)=\left(\mathcal{G}_{-}^{a_{j}}\right)^{\prime}(s) & {\left[\varphi^{\prime} \circ \mathcal{G}_{-}^{a_{j}}(s)-\varphi^{\prime} \circ \mathcal{G}_{-}^{a_{j}}\left(\sum_{l^{\prime}=0}^{l-1}(-1)^{l^{\prime}} d_{\tilde{\jmath}-l^{\prime}-1}+s\right)\right.} \\
& \left.+\varphi^{\prime} \circ \mathcal{G}_{-}^{a_{j}}\left(s-d_{\tilde{\jmath}-l}\right)-\varphi^{\prime} \circ \mathcal{G}_{-}^{a_{j}}\left(\mathcal{G}_{-}^{a_{j}}(s)-d_{\tilde{\jmath}-l}+\mathcal{G}_{+}^{a_{j}}\left(d_{\tilde{\jmath}-l-1}\right)\right)\right] \\
& +\left(1-\left(\mathcal{G}_{-}^{a_{j}}\right)^{\prime}(s)\right)\left[\varphi^{\prime} \circ \mathcal{G}_{-}^{a_{j}}\left(s-d_{\tilde{\jmath}-l}\right)-\varphi^{\prime} \circ \mathcal{G}_{-}^{a_{j}}\left(\sum_{l^{\prime}=0}^{l-1}(-1)^{l^{\prime}} d_{\tilde{\jmath}-l^{\prime}-1}+s\right)\right] .
\end{aligned}
$$

One has $0 \leq \sum_{l^{\prime}=0}^{l^{\prime \prime}}(-1)^{l^{\prime}} d_{\tilde{\jmath}-l^{\prime}-1} \leq b_{j}$ for $l^{\prime \prime}=l-1, l-2$, and $s-\mathcal{G}_{-}^{a_{j}}(s)-\mathcal{G}_{+}^{a_{j}}\left(d_{\tilde{\jmath}-l-1}\right)=$ $\mathcal{G}_{+}^{a_{j}}(s)-\mathcal{G}_{+}^{\overline{a_{j}}}\left(d_{\tilde{\jmath}-l-1}\right) \leq 0$ for $s \in\left[d_{\tilde{\jmath}-l}, d_{\tilde{\jmath}-l-1}\right]$. Therefore, $\psi_{j, l}(s)$ is a sum of nonpositive terms and $r_{\tilde{\jmath}-l} \leq 0$ for any $\varphi$.

$\mathrm{RR} \mathrm{n}^{\circ} 3443$ 
Using the fact that in the expression of $r_{\tilde{\jmath}-l}, \varphi^{\prime}$ is applied to numbers $s \in\left[0, c_{j}\right]$, one obtains for $\varphi(s)=s^{\gamma+1}$ :

$$
\begin{aligned}
r_{\tilde{\jmath}-l} \leq & -\gamma(\gamma+1) \lambda^{2} c_{j}^{\gamma-1}\left[\left(\sum_{l^{\prime}=0}^{l-1}(-1)^{l^{\prime}} d_{\tilde{\jmath}-l^{\prime}-1}\right)\left(d_{\tilde{\jmath}-l-1}-d_{\tilde{\jmath}-l}\right)\right. \\
& \left.+\frac{\lambda}{2}\left(d_{\tilde{\jmath}-l-1}-d_{\tilde{\jmath}-l}\right)^{2}+\left(\sum_{l^{\prime}=0}^{l-2}(-1)^{l^{\prime}} d_{\tilde{\jmath}-l^{\prime}-1}\right)\left(d_{\tilde{\jmath}-l-1}-d_{\tilde{\jmath}-l}\right)\right] \\
\leq & -\gamma(\gamma+1) \lambda^{2} c_{j}^{\gamma-1}\left[\frac{\lambda}{2}\left(d_{\tilde{\jmath}-l-1}-d_{\tilde{\jmath}-l}\right)^{2}+\left(\sum_{l^{\prime}=0}^{l-2}(-1)^{l^{\prime}} d_{\tilde{\jmath}-l^{\prime}-1}\right)\left(d_{\tilde{\jmath}-l-1}-d_{\tilde{\jmath}-l}\right)\right] \\
\leq & -\gamma(\gamma+1) \frac{\lambda^{3}}{2} c_{j}^{\gamma-1}\left[\left(\sum_{l^{\prime}=0}^{l}(-1)^{l^{\prime}} d_{\tilde{\jmath}-l^{\prime}-1}\right)^{2}-\left(\sum_{l^{\prime}=0}^{l-2}(-1)^{l^{\prime}} d_{\tilde{\jmath}-l^{\prime}-1}\right)^{2}\right],
\end{aligned}
$$

as $\lambda<1$.

- Summing the last inequality over all nonzero even indexes, one obtains

$$
\begin{aligned}
\sum_{l=1}^{\bar{l}} r_{\tilde{\jmath}-2 l} & \leq-\gamma(\gamma+1) \frac{\lambda^{3}}{2} c_{j}^{\gamma-1}\left(b_{j}^{2}-d_{\tilde{\jmath}-1}^{2}\right) \\
& \leq-\gamma(\gamma+1) \frac{\lambda^{3}}{2} c_{j}^{\gamma-1}\left(b_{j}-d_{\tilde{\jmath}-1}\right)^{2} \\
& \leq-\gamma(\gamma+1) \frac{\lambda^{3}}{2(1-\lambda)^{2}} c_{j}^{\gamma-1}\left(c_{j}-c_{j+\frac{1}{3}}\right)^{2}
\end{aligned}
$$

that is $(25)$.

- At last, summing the contributions over all nonzero indexes, one gets:

$$
\begin{aligned}
\sum_{l=1}^{2 \bar{l}} r_{\tilde{\jmath}-l} \leq & -\gamma(\gamma+1) \frac{\lambda^{3}}{2} c_{j}^{\gamma-1}\left(b_{j}^{2}-d_{\tilde{\jmath}-1}^{2}+\left(d_{\tilde{\jmath}-1}^{2}-d_{\tilde{\jmath}-2}^{2}\right)\right. \\
& \left.+\left(d_{\tilde{\jmath}-3}^{2}-d_{\tilde{\jmath}-4}^{2}\right)+\cdots+\left(d_{\tilde{\jmath}-2 \bar{l}+1}^{2}-d_{\tilde{\jmath}-2 \bar{l}}^{2}\right)\right) \\
\leq & -\gamma(\gamma+1) \frac{\lambda^{3}}{2} c_{j}^{\gamma-1}\left(b_{j}^{2}-d_{\tilde{\jmath}-2 \bar{l}-1}^{2}\right), \quad \text { as } d_{\tilde{\jmath}-2 l-1} \geq d_{\tilde{\jmath}-2 l} \text { for } l=1, \ldots \bar{l} . \\
\leq & -\gamma(\gamma+1) \frac{\lambda^{3}}{2(1-\lambda)^{2}} c_{j}^{\gamma-1}\left(c_{j}-c_{j-\frac{1}{3}}\right)^{2},
\end{aligned}
$$

that is (26).

INRIA 


\subsection{Proof of Proposition 9 for general $I$ such that $I$ does not contain accumulation points of $Z$}

One studies here the case where $I \cap \operatorname{Acc} Z=\emptyset$, but $I \cap(\operatorname{Acc} Z+h) \neq \emptyset$. The same notations as in Sections 4.3 and 4.4 are used for $U, \tilde{U}, U_{j}, b_{j}, \tilde{X}, X^{\prime}$.

Since $I-h$ contains accumulation points of $Z$, the connected components $\tilde{U}_{i}, i \in \mathcal{I} \subset \mathbb{N}$ of $\tilde{U}$ cannot be ordered in increasing order; to recall this fact, one uses a different notation of the indexes, namely $i \in \mathcal{I}$ instead of $j \in \mathcal{J}$. They still correspond to the maximal intervals on which $\tilde{X}$ is monotonous.

Let $\varepsilon>0$. There exists a finite subset $\mathcal{I}_{f}$ of $\mathcal{I}$ such that

$$
\sum_{i \in \mathcal{I} \backslash \mathcal{I}_{f}} \mu_{\varphi}\left(\tilde{U}_{i}\right) \leq \varphi\left(\operatorname{meas} \tilde{U}-\sum_{i \in \mathcal{I}_{f}} \text { meas } \tilde{U}_{i}\right) \leq \varepsilon .
$$

Let us approximate $\tilde{X}$ by another solution $\tilde{X}^{\varepsilon}$ of (1)-(5), defined as follows:

$$
\tilde{X}^{\varepsilon}=\tilde{X} \text { on } \bigcup_{i \in \mathcal{I}_{f}} \tilde{U}_{i}, \quad \tilde{X}^{\varepsilon}=X^{\prime} \text { on } U_{j} \text { such that } U_{j} \cap\left(\bigcup_{i \in \mathcal{I}_{f}} \tilde{U}_{i}\right)=\emptyset,
$$

and, to complete the definition, on any connected component of $U_{j} \backslash \bigcup_{i \in \mathcal{I}_{f}} \tilde{U}_{i}$, one chooses $\tilde{X}^{\varepsilon}$ increasing and then decreasing if $j$ is even, decreasing and then increasing if $j$ is odd. The function $\tilde{X}^{\varepsilon}$ verifies (18) for a new, ordered, sequence $\tilde{U}_{j}^{\varepsilon}$, of lengths $\tilde{b}_{j}^{\varepsilon}$. For this sequence, the result of Proposition 9 applies, due to Section 4.4, and gives:

$$
\mu_{\varphi}\left(\tilde{b}^{\varepsilon}\right) \leq \mu_{\varphi}(b) \text { for any } \varphi, \quad \mu_{\gamma+1}\left(\tilde{b}^{\varepsilon}\right) \leq \mu_{\gamma+1}(b)-\theta \frac{\|b\|_{p}^{\frac{3}{p-1}+2+\gamma}}{\|b\|_{1}^{\frac{3}{p-1}+1}} .
$$

In order to get the result of Proposition 9 for $I$, it suffices to prove that for any $\varphi$,

$$
\mu_{\varphi}(I-h) \leq \limsup _{\varepsilon \rightarrow 0^{+}} \mu_{\varphi}\left(\tilde{b}^{\varepsilon}\right) .
$$

One has

$$
\mu_{\varphi}(I-h)=\sum_{i \in \mathcal{I}} \mu_{\varphi}\left(\tilde{U}_{i}\right)=\sum_{i \in \mathcal{I}_{f}} \mu_{\varphi}\left(\tilde{U}_{i}\right)+\sum_{i \in \mathcal{I} \backslash \mathcal{I}_{f}} \mu_{\varphi}\left(\tilde{U}_{i}\right)
$$

Now, for any $\varepsilon>0$,

$$
\sum_{i \in \mathcal{I}_{f}} \mu_{\varphi}\left(\tilde{U}_{i}\right) \leq \mu_{\varphi}\left(\tilde{b}^{\varepsilon}\right)
$$

since any $\tilde{U}_{i}$ with $i \in \mathcal{I}_{f}$ is included in a set $\tilde{U}_{j}^{\varepsilon}$, and any $\tilde{U}_{j}^{\varepsilon}$ contains at most one $\tilde{U}_{i}$ with $i \in \mathcal{I}_{f}$. Formula (27) permits to conclude.

$\mathrm{RR} \mathrm{n}^{\circ} 3443$ 


\subsection{Proof of Proposition 9 for general $I$}

Let now $I$ be a general open interval with bounds in Acc $Z$ and meas $I \leq h$, and let $U=I \backslash$ Acc $Z$. The set $U$ is open, and is then the (finite or countable) union of its connected components $U_{i}, i \in \mathcal{I} \subset \mathbb{N}$, which form a partition of $U$. However in general, the sets $U_{i}$ cannot be ordered. Since meas $Z=0$, one has:

$$
\sum_{i \in \mathcal{I}} \text { meas } U_{i}=\text { meas } U=\text { meas } I \text {. }
$$

Moreover, $I \backslash Z=U \backslash Z=\bigcup_{i \in \mathcal{I}}\left(U_{i} \backslash Z\right)$ (disjoint union) and, by the definition of $\mu_{\varphi}$,

$$
\mu_{\varphi}(I)=\sum_{i \in \mathcal{I}} \mu_{\varphi}\left(U_{i}\right)
$$

Since $t \in \operatorname{Acc} Z$ implies $t-h \in \operatorname{Acc} Z$ (by Lemma 7), then $(I-h) \backslash \operatorname{Acc} Z \subset U-h$ and $(I-h) \backslash Z=(U-h) \backslash Z=\bigcup_{i \in \mathcal{I}}\left(\left(U_{i}-h\right) \backslash Z\right)$ (disjoint union). Therefore,

$$
\mu_{\varphi}(I-h)=\sum_{i \in \mathcal{I}} \mu_{\varphi}\left(U_{i}-h\right) .
$$

Since Proposition 9 has already been proved for open intervals with bounds in Acc $Z$ and no accumulation points of $Z$ inside, one has, for any $i \in \mathcal{I}$ :

$$
\begin{gathered}
\mu_{\varphi}\left(U_{i}-h\right) \leq \mu_{\varphi}\left(U_{i}\right) \\
\mu_{\gamma+1}\left(U_{i}-h\right) \leq \mu_{\gamma+1}\left(U_{i}\right)-\theta \frac{\left\|U_{i}\right\|_{p}^{\frac{3}{p-1}+2+\gamma}}{\left(\operatorname{meas} U_{i}\right)^{\frac{3}{p-1}+1}} .
\end{gathered}
$$

Summing the previous inequalities, one obtains (16), and:

$$
\mu_{\varphi}(I-h) \leq \mu_{\varphi}(I)-\theta \sum_{i \in \mathcal{I}} \frac{\left\|U_{i}\right\|_{p}^{\frac{3}{p-1}+2+\gamma}}{\left(\operatorname{meas} U_{i}\right)^{\frac{3}{p-1}+1}} .
$$

Now, let us prove that

$$
\sum_{i \in \mathcal{I}} \frac{\left\|U_{i}\right\|_{p}^{\frac{3}{p-1}+2+\gamma}}{\left(\operatorname{meas} U_{i}\right)^{\frac{3}{p-1}+1}} \geq \frac{\|I\|_{p}^{\frac{3}{p-1}+2+\gamma}}{(\text { meas } I)^{\frac{3}{p-1}+1}} .
$$


Indeed, let $q \triangleq\left(\frac{3}{p-1}+1\right) p /\left(\frac{3}{p-1}+2+\gamma\right)$, then $q \geq 1$ since $\gamma \leq 1$ and $p \geq 1$. Therefore

$$
\begin{aligned}
\|I\|_{p}^{\frac{3}{p-1}+2+\gamma} & =\left(\|I\|_{p}^{p}\right)^{\frac{1}{q}\left(\frac{3}{p-1}+1\right)}=\left(\sum_{i \in \mathcal{I}}\left\|U_{i}\right\|_{p}^{p}\right)^{\frac{1}{q}\left(\frac{3}{p-1}+1\right)} \\
& \leq \max _{i \in \mathcal{I}}\left(\frac{\left\|U_{i}\right\|_{p}^{p}}{\left(\operatorname{meas} U_{i}\right)^{q}}\right)^{\frac{1}{q}\left(\frac{3}{p-1}+1\right)}\left(\sum_{i \in \mathcal{I}}\left(\operatorname{meas} U_{i}\right)^{q}\right)^{\frac{1}{q}\left(\frac{3}{p-1}+1\right)} \\
& \leq \sum_{i \in \mathcal{I}} \frac{\left\|U_{i}\right\|_{p}^{\frac{3}{p-1}+2+\gamma}}{\left(\operatorname{meas} U_{i}\right)^{\frac{3}{p-1}+1}}(\operatorname{meas} I)^{\frac{3}{p-1}+1} .
\end{aligned}
$$

This ends the proof of (17) for general $I$ with bounds in Acc $Z$, and achieves the proof of Proposition 9.

\subsection{A proof using Schur convexity when $F$ is odd}

When $F$ is odd, then

$$
\mathcal{G}_{ \pm}^{P}=\mathcal{G}_{ \pm}^{N}, \quad \varphi^{P}=\varphi^{N} .
$$

In this section, one proves that $\mu_{\varphi}(I-h) \leq \mu_{\varphi}\left(b^{\prime}\right)$ for all $I$ such that $I \cap \operatorname{Acc} Z=\emptyset$, which implies Proposition 9, due to Section 4.3. This proof replaces that of Sections 4.4 and 4.5.

Let us first study, as in Section 4.4, the case where $I \cap \operatorname{Acc} Z=I \cap(\operatorname{Acc} Z+h)=\emptyset$ and $\tilde{b} \neq b^{\prime}$.

Let $U, U_{j}, b_{j}, \tilde{U}, \tilde{U}_{j}, \tilde{b}_{j}, X^{\prime}$ be defined as in Section 4.3 , and $\tilde{X}, \tilde{\jmath}=\tilde{\jmath}(j), \bar{l}=\bar{l}(j)$ as in Section 4.4. For any $j \in \mathbb{Z}$, let $T_{j}$ be the following transformation on the function $\tilde{X}$ (see Figure 3):

$$
T_{j} \tilde{X}=\tilde{X} \text { on } I \backslash U_{j}, \quad T_{j} \tilde{X}=X^{\prime} \text { on } U_{j} .
$$

Let us partition $U_{j}$ into two subintervals $U_{j}^{-}$and $U_{j}^{+}$, with $U_{j}^{-} \leq U_{j}^{+}$and

$$
\text { meas } U_{j}^{-}=b_{j}^{-} \triangleq \mathcal{G}_{-}^{P}\left(b_{j}\right), \quad \text { meas } U_{j}^{+}=b_{j}^{+} \triangleq b_{j}-b_{j}^{-}=\mathcal{G}_{+}^{P}\left(b_{j}\right) \text {. }
$$

If $\tilde{X}$ is equal to $X^{\prime}$ on $U_{j}$, then $\bar{l}=0$ and

$$
\tilde{U}_{\tilde{\jmath}-1} \cap U_{j}=U_{j}^{-}, \quad \tilde{U}_{\tilde{\jmath}} \cap U_{j}=U_{j}^{+} .
$$

The transformation $T_{j}$ generates the following transformation, also denoted $T_{j}$, on $\tilde{U}$ and $\tilde{b}$. The sets $\tilde{U}_{\tilde{\jmath}-2 \bar{l}-1}, \ldots, \tilde{U}_{\tilde{\jmath}}$ are replaced by $\left(T_{j} \tilde{U}\right)_{\tilde{\jmath}-1}=\tilde{U}_{\tilde{\jmath}-2 \bar{l}-1} \cup U_{j}^{-}$and $\left(T_{j} \tilde{U}\right)_{\tilde{\jmath}}=$ 
$\tilde{U}_{\tilde{\jmath}} \cup U_{j}^{+}$. Therefore, the numbers $\tilde{b}_{\tilde{\jmath}-2 \bar{l}-1}, \ldots, \tilde{b}_{\tilde{\jmath}}$ are replaced by $\left(T_{j} \tilde{b}\right)_{\tilde{\jmath}-1}=b^{-}+b_{j}^{-}$and $\left(T_{j} \tilde{b}\right)_{\tilde{\jmath}}=b^{+}+b_{j}^{+}$, with

$$
b^{-\triangleq} \text { meas } \tilde{U}_{\tilde{\jmath}-2 \bar{l}-1} \backslash U_{j}, \quad b^{+} \triangleq \text { meas } \tilde{U}_{\tilde{\jmath}} \backslash U_{j} .
$$

The other components remain unchanged, up to a reindexation.

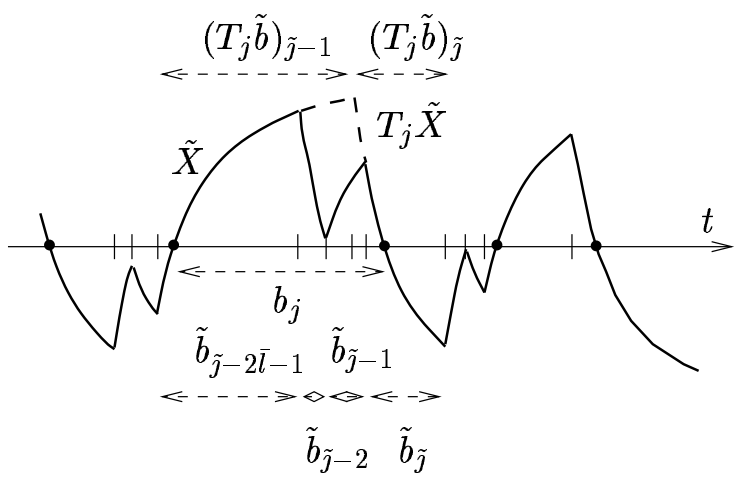

Figure 3: Suboptimal predecessors and their transformation $(j$ is even, $\bar{l}(j)=1)$

Lemma 12. For all predecessor $\tilde{b}$ of $b$ and for all $j \in \mathbb{Z}$, one has

$$
\mu_{\varphi}(\tilde{b}) \leq \mu_{\varphi}\left(T_{j} \tilde{b}\right) .
$$

Proof. Let us consider the two $(2 \bar{l}+2)$-dimensional vectors

$$
u \triangleq\left(\tilde{b}_{\tilde{\jmath}-2 \bar{l}-1}, \ldots, \tilde{b}_{\tilde{\jmath}}\right), \quad v \triangleq\left(0, \ldots, 0,\left(T_{j} \tilde{b}\right)_{\tilde{\jmath}-1},\left(T_{j} \tilde{b}\right)_{\tilde{\jmath}}\right) .
$$

Since $T_{j} \tilde{b}$ and $\tilde{b}$ differ only (up to a reindexation) by the coordinates present in $u$ and $v$, one has

$$
\mu_{\varphi}\left(T_{j} \tilde{b}\right)-\mu_{\varphi}(\tilde{b})=\sum_{l=1}^{2 \bar{l}+2} \varphi^{P}\left(v_{l}\right)-\sum_{l=1}^{2 \bar{l}+2} \varphi^{P}\left(u_{l}\right) .
$$

The proof of Lemma 12 then reduces to show $g(u) \leq g(v)$ for $g: \mathbb{R}^{2 \bar{l}+2} \rightarrow \mathbb{R}, u \mapsto g(u)=$ $\sum_{l=1}^{2 \bar{l}+2} \varphi^{P}\left(u_{l}\right)$. Since $\varphi^{P}$ is a convex function, $g$ is Schur-convex (see [16], [9], and [12, Proposition 3.C.1, p. 64], where a general presentation of the subject may be found). In other words, $g(u) \leq g(v)$ for any $u, v \in \mathbb{R}^{2 \bar{l}+2}$ such that $u$ is majorized by $v$, that is fulfilling 
the two following conditions:

$$
\begin{gathered}
\sum_{l=1}^{2 \bar{l}+2} u_{l}=\sum_{l=1}^{2 \bar{l}+2} v_{l}, \\
\max _{\mathcal{L} \subset\{1, \ldots, 2 \bar{L}+2\}}^{\operatorname{card}=L} \underset{l}{L} u_{l \in \mathcal{L}} u_{l} \leq \max _{\substack{\operatorname{card} \mathcal{L}=L \\
\mathcal{L} \subset\{1, \ldots, 2 l+2\}}} \sum_{l \in \mathcal{L}} v_{l}, \quad \forall L=1, \ldots, 2 \bar{l}+2 .
\end{gathered}
$$

It then suffices to prove that $v$ majorizes $u$, for $u, v$ given by (29).

Since

$$
\sum_{j^{\prime}=-\infty}^{+\infty} \tilde{b}_{j^{\prime}}=\sum_{j^{\prime}=-\infty}^{+\infty}\left(T_{j} \tilde{b}\right)_{j^{\prime}}=\sum_{j=-\infty}^{+\infty} b_{j}
$$

one has $\sum_{l=1}^{2 \bar{l}+2} u_{l}=\sum_{l=1}^{2 \bar{l}+2} v_{l}$, that is (30). Since $v$ has only two nonzero coordinates, (31) is fulfilled for $L \geq 2$. The case $L=1$ is equivalent to

$$
\max _{l=0, \ldots, 2 \bar{l}+1} \tilde{b}_{\tilde{\jmath}-l} \leq \max \left\{\left(T_{j} \tilde{b}\right)_{\tilde{\jmath}-1},\left(T_{j} \tilde{b}\right)_{\tilde{\jmath}}\right\}
$$

From the previous computations and as $b^{+}, b^{-} \geq 0$, it is sufficient to prove

$$
\begin{gathered}
\max \left\{\tilde{b}_{\tilde{\jmath}-2 \bar{l}-1}-b^{-}, \tilde{b}_{\tilde{\jmath}-2 \bar{l}+1}, \ldots, \tilde{b}_{\tilde{\jmath}-1}\right\} \leq b_{j}^{-}, \\
\quad \max \left\{\tilde{b}_{\tilde{\jmath}-2 \bar{l}}, \ldots, \tilde{b}_{\tilde{\jmath}-2}, \tilde{b}_{\tilde{\jmath}}-b^{+}\right\} \leq b_{j}^{+},
\end{gathered}
$$

that is

$$
\left\{\begin{array}{l}
\max _{l=1, \ldots 2 \bar{l}+1} \text { meas } \tilde{U}_{\tilde{\jmath}-l} \cap U_{j} \leq \operatorname{meas} U_{j}^{-} \\
\begin{array}{c}
l \text { odd } \\
l=0, \ldots 2 \bar{l} \\
l \text { even }
\end{array}
\end{array}\right.
$$

Since the intervals $\tilde{U}_{l}$ are the maximal intervals on which $\tilde{X}$ is monotonous, the previous inequalities mean that the maximum length of a subinterval of $U_{j}$ on which $\tilde{X}$ increases (resp. decreases) is less than the length of the (unique) subinterval of $U_{j}$ on which $X^{\prime}$ increases (resp. decreases), that is $b_{j}^{-}$(resp. $b_{j}^{+}$) if $j$ is even, $b_{j}^{+}$(resp. $b_{j}^{-}$) if $j$ is odd. It is easy to see from Figure 3 that this holds. This is indeed a consequence of the stationnarity of the equation $\dot{X}=\varepsilon+F(X)(\varepsilon= \pm 1)$.

For instance, let us suppose that $j$ is even, and consider an interval $\left(t^{\prime}, t^{\prime \prime}\right) \subset U_{j}=$ $\left(t, t+b_{j}\right)$ where $\tilde{X}$ increases. Then $\tilde{X}$ satisfies $\dot{\tilde{X}}=1+F(\tilde{X})$ on $\left(t^{\prime}, t^{\prime \prime}\right)$, and $\tilde{X}\left(t^{\prime}\right) \geq 0$. But $X^{\prime}$ satisfies the same equation on $\left(t, t+b_{j}^{-}\right)$, and $X^{\prime}(t)=0$. If $t^{\prime \prime}-t^{\prime}>b_{j}^{-}$, then $\tilde{X}\left(t^{\prime \prime}\right)>X^{\prime}\left(t+b_{j}^{-}\right)$(by the stationnarity of $\dot{X}=1+F(X)$ and the fact that $X^{\prime}$ and 
$\tilde{X}$ increase), and $t^{\prime \prime}>t+b_{j}^{-}$. Using all the properties of $X^{\prime}$ and $\tilde{X}$, that is: $X^{\prime}$ verifies $\dot{X}^{\prime}=-1+F\left(X^{\prime}\right)$ on $\left(t+b_{j}^{-}, t+b_{j}\right), \tilde{X}$ either increases or is a solution of the same equation on $\left(t^{\prime \prime}, t+b_{j}\right), \tilde{X}\left(t^{\prime \prime}\right)>X^{\prime}\left(t+b_{j}^{-}\right)$and $\tilde{X}\left(t+b_{j}\right)=X^{\prime}\left(t+b_{j}\right)=0$, one obtains that the trajectory of $\tilde{X}$ on $\left[t^{\prime \prime}, t+b_{j}\right]$ contains all the trajectory of $X^{\prime}$ on $\left[t+b_{j}^{-}, t+b_{j}\right]$ but at different instants. This is impossible, since there is not enough time: $t+b_{j}-t^{\prime \prime}<\left(t+b_{j}\right)-\left(t+b_{j}^{-}\right)$.

From this, one deduces (32). Analogously, one may show (33). This achieves the proof of Lemma 12.

By applying the transformations $T_{j}$ successively for $j \in\{-m, \ldots, m\}$, one obtains a function $\tilde{X}^{m} \triangleq\left(T_{-m} \circ \cdots \circ T_{m}\right) \tilde{X}$ (the composition is commutative), equal to $X^{\prime}$ on the closure $\mathrm{cl}\left(\bigcup_{j=-m}^{m} U_{j}\right)$. Since the limit of the latter increasing sequence of intervals is equal to $I, \tilde{X}^{m}$ tends to $X^{\prime}$ (pointwise). Applying the following lemma to $\tilde{b}^{m}=\left(T_{-m} \circ \cdots \circ T_{m}\right) \tilde{b}$, one obtains $\mu_{\varphi}\left(\tilde{b}^{m}\right) \rightarrow \mu_{\varphi}\left(b^{\prime}\right)$ when $m \rightarrow+\infty$, and finally deduces, with the help of Lemma 12 , that $\mu_{\varphi}(\tilde{b}) \leq \mu_{\varphi}\left(b^{\prime}\right)$. Therefore, $b^{\prime}$ is an optimal predecessor, in the sense that

$$
\mu_{\varphi}\left(b^{\prime}\right)=\max _{\tilde{b} \text { predecessor of } b} \mu_{\varphi}(\tilde{b}),
$$

and (17) is proved for general $I$ such that $I$ and $I-h$ do not contain accumulation points of $Z$.

Lemma 13. Let $\tilde{b}$ be a predecessor of $b$ and $\tilde{X}$ satisfy (18). If $\tilde{X}=X^{\prime}$ on the closure $\operatorname{cl}\left(\bigcup_{j=-m}^{m} U_{j}\right)$, then

$$
\left|\mu_{\varphi}(\tilde{b})-\mu_{\varphi}\left(b^{\prime}\right)\right| \leq \frac{\varphi^{P}(h)}{h} \operatorname{meas}\left(I \backslash \bigcup_{j=-m+1}^{m-1} U_{j}\right)=\frac{\varphi^{P}(h)}{h}\left(\sum_{j=-\infty}^{-m} b_{j}+\sum_{j=m}^{+\infty} b_{j}\right) .
$$

Proof. The proof proceeds from the following inequalities:

$$
\begin{aligned}
\left|\mu_{\varphi}(\tilde{b})-\mu_{\varphi}\left(b^{\prime}\right)\right| \leq \mid & \left|\sum_{j \in \tilde{\mathcal{J}}_{m}} \varphi^{P}\left(\tilde{b}_{j}\right)-\sum_{j \in \mathcal{J}_{m}^{\prime}} \varphi^{P}\left(b_{j}^{\prime}\right)\right| \\
& \text { where } \tilde{\mathcal{J}}_{m} \triangleq\left\{j \in \mathbb{Z}: \tilde{U}_{j} \not \subset \mathrm{cl}\left(\bigcup_{l=-m}^{m} U_{l}\right)\right\} \text { and similarly for } \mathcal{J}_{m}^{\prime} \\
\leq & \frac{\varphi^{P}(h)}{h} \max \left\{\sum_{j \in \tilde{\mathcal{J}}_{m}} \tilde{b}_{j}, \sum_{j \in \mathcal{J}_{m}^{\prime}} b_{j}^{\prime}\right\} \\
\leq & \frac{\varphi^{P}(h)}{h}\left(\sum_{j=-\infty}^{-m} b_{j}+\sum_{j=m}^{+\infty} b_{j}\right)
\end{aligned}
$$

INRIA 
using the fact that $\varphi^{P}(s) \leq s \varphi^{P}(h) / h$ for $0 \leq s \leq h$ (due to convexity of $\varphi^{P}$ and $\varphi^{P}(0)=0$ ) and the definition of $\tilde{\mathcal{J}}_{m}, \mathcal{J}_{m}^{\prime}$.

We now consider the case where $I \cap(\operatorname{Acc} Z+h) \neq \emptyset$. We get from Section 4.5 that

$$
\mu_{\varphi}(I-h) \leq \liminf _{\varepsilon \rightarrow 0^{+}} \mu_{\varphi}\left(\tilde{b}^{\varepsilon}\right),
$$

where the $\tilde{b}^{\varepsilon}$ are predecessors of $b$. From what was proved in the present section, we hence deduce $\mu_{\varphi}(I-h) \leq \mu_{\varphi}\left(b^{\prime}\right)$.

Remark that one may also generalize Lemma 12 to this case. Indeed, the weaker order relation induced by (31), called weak majorization, suffices to prove $g(u) \leq g(v)$, since $\varphi^{P}$ is increasing in $\mathbb{R}^{+}$and $u_{l}, v_{l} \geq 0$; this result is proved in $[19,20]$ and may be found in $[12$, 3.C.1.b, p. 64]. The weak majorization is satisfied by the vectors $u$ and $v$ generated by some finite subsets of measures of those $\operatorname{cl}\left(\tilde{U}_{i}\right)$ and $\left(T_{j} \tilde{U}\right)_{i}$ which intersect $\operatorname{cl}\left(U_{j}\right)$. Then, Lemma 12 is deduced by passing to the limit.

\section{A Appendix - Proof of Lemma 10}

In a first step, one uses Gagliardo-Nirenberg result $[8,13]$ under the following form: for any $f \in L^{1}(\mathbb{R}) \cap L^{p}(\mathbb{R})$ such that $f^{\prime} \in L^{q}(\mathbb{R})$, one has

$$
\|f\|_{1}^{\frac{p q-p+q}{p-1}}\left\|f^{\prime}\right\|_{q}^{q} \geq\left(\frac{q}{2 q-1}\right)^{q}\|f\|^{\frac{p(2 q-1)}{p-1}} .
$$

For sake of completness, one provides the proof of (34). Let us first prove that $\lim _{b \rightarrow-\infty} f(b)$ exists - and hence is 0 . For any $s^{\prime}, s^{\prime \prime} \in \mathbb{R}$, one has (by Hölder inequality):

$\frac{q}{2 q-1}\left|\left[|f(s)|^{\frac{2 q-1}{q}}\right]_{s^{\prime}}^{s^{\prime \prime}}\right| \leq \int_{s^{\prime}}^{s^{\prime \prime}}\left|f^{\prime}(s)\right||f(s)|^{\frac{q-1}{q}} d s \leq\left(\int_{s^{\prime}}^{s^{\prime \prime}}\left|f^{\prime}(s)\right|^{q} d s\right)^{\frac{1}{q}}\left(\int_{s^{\prime}}^{s^{\prime \prime}}|f(s)| d s\right)^{\frac{q-1}{q}}$, 
which tends to 0 when $\max \left\{s^{\prime}, s^{\prime \prime}\right\} \rightarrow-\infty$. From the following inequalities:

$$
\begin{aligned}
\|f\|_{p}^{p} & =\int_{\mathbb{R}}|f(s)|^{p} d s=\int_{\mathbb{R}}|f(s)||f(s)|^{p-1} d s \\
& =\int_{\mathbb{R}}|f(s)|\left(\int_{-\infty}^{s}\left(|f|^{\frac{2 q-1}{q}}\right)^{\prime}\left(s^{\prime}\right) d s^{\prime}\right)^{\frac{q(p-1)}{2 q-1}} d s \\
& =\left(\frac{2 q-1}{q}\right)^{\frac{q(p-1)}{2 q-1}} \int_{\mathbb{R}}|f(s)|\left(\int_{-\infty}^{s} f^{\prime}\left(s^{\prime}\right) \operatorname{sgn} f\left(s^{\prime}\right)\left|f\left(s^{\prime}\right)\right|^{\frac{q-1}{q}} d s^{\prime}\right)^{\frac{q(p-1)}{2 q-1}} d s \\
& \leq\left(\frac{2 q-1}{q}\right)^{\frac{q(p-1)}{2 q-1}} \int_{\mathbb{R}}|f(s)| d s\left(\left(\int_{\mathbb{R}}\left|f^{\prime}(s)\right|^{q} d s\right)^{\frac{1}{q}}\left(\int_{\mathbb{R}}|f(s)| d s\right)^{\frac{q-1}{q}}\right)^{\frac{q(p-1)}{2 q-1}}
\end{aligned}
$$

(by Hölder inequality)

$$
=\left(\frac{2 q-1}{q}\right)^{\frac{q(p-1)}{2 q-1}}\|f\|_{1}^{\frac{p q-p+q}{2 q-1}}\left\|f^{\prime}\right\|_{q^{\frac{q(p-1)}{2 q-1}}}
$$

one gets (34) when $p<+\infty$. Inequality (34) holds for $p=+\infty$ too, by passing to the limit.

Now, let us define $f \in W^{1, \infty}(\mathbb{R})$ by:

$$
f(s)=\left(b_{j+1}-b_{j}\right) s+(j+1) b_{j}-j b_{j+1} \text { for } s \in[j, j+1) .
$$

One proves easily that $\|f\|_{1} \leq\|b\|_{1},\left\|f^{\prime}\right\|_{q}=\left\|b_{j+1}-b_{j}\right\|_{q}$. Moreover,

$$
\begin{aligned}
\|f\|_{p}^{p} & =\frac{1}{p+1} \sum_{j \in \mathbb{Z}} \frac{b_{j+1}\left|b_{j+1}\right|^{p}-b_{j}\left|b_{j}\right|^{p}}{b_{j+1}-b_{j}} \\
& =\frac{1}{2(p+1)} \sum_{j \in \mathbb{Z}}\left(\left|b_{j+1}\right|^{p}+\left|b_{j}\right|^{p}+\frac{b_{j+1}+b_{j}}{b_{j+1}-b_{j}}\left(\left|b_{j+1}\right|^{p}-\left|b_{j}\right|^{p}\right)\right) \\
& \geq \frac{1}{2(p+1)} \sum_{j \in \mathbb{Z}}\left(\left|b_{j+1}\right|^{p}+\left|b_{j}\right|^{p}\right)=\frac{1}{p+1}\left\|b_{j}\right\|_{p}^{p} .
\end{aligned}
$$

This achieves the proof of Lemma 10 in the case $p<+\infty$, with

$$
C_{p, q}=\left(\frac{q}{2 q-1}\right)^{q}\left(\frac{1}{p+1}\right)^{\frac{2 q-1}{p-1}} .
$$

Since

$$
\lim _{p \rightarrow+\infty} C_{p, q}=\left(\frac{q}{2 q-1}\right)^{q}
$$

Lemma 10 also holds for $p=+\infty$, with $C_{\infty, q}$ equal to this limit.

INRIA 


\section{References}

[1] M. Akian, P.-A. Bliman, M. Sorine, P.I. control of nonlinear oscillations for a system with delay, Rapport de Recherche 3422, INRIA, May 1998.

[2] U. An der Heiden, K. Reichard, Multitude of oscillatory behaviour in a nonlinear second order differential-difference equation, Z. angew. Math. Mech., 706 (1990), T621-T624.

[3] O. Diekmann, S.A. van Gils, S.M. Verduyn Lunel, H.-O. Walther, "Delay equations: functional-, complex- and nonlinear analysis", Applied Mathematical Sciences 110, Springer-Verlag, New-York, 1995.

[4] J.G. Dix, Infinite frequency solutions to discontinuous control systems with variable delay, to appear in Israel J. of Math.

[5] G.F. Franklin, J.D. Powell, A. Emami-Naeini, "Feedback control of dynamic systems", Addison-Wesley Publishing Company, 1988.

[6] L. Fridman, E. Shustin, E. Fridman, Steady modes and sliding modes in the relay control systems with time delay, Proc. 35th Conf. on Decision and Control, Kobe, Japan (1996), 4601-4606.

[7] L. Fridman, E. Fridman, E. Shustin, The phenomenon of the second order steady modes in the relay control systems with time delay, Proc. of the 4 th European Control Conference, Brussels, Belgium (1997).

[8] E. Gagliardo. Ulteriori proprieta di alcune classi di funzioni in piu variabili, Ricerche di Mat. 8 (1959), 24-51.

[9] G.H. Hardy, J.E. Littlewood, G. Pólya, Some simple inequalities satisfied by convex functions, Messenger Math. 58 (1929), 145-152

[10] A.F. Ivanov, J. Losson, Stable rapidly oscillating solutions in delay differential equations with negative feedback, Cadsem Report 97-004, Deakin University, Australia, January 1997.

[11] J. Mallet-Paret, Morse decompositions for delay-differential equations, Journal of differential equations 72 (1988), 270-315.

[12] A.W. Marshall, I. Olkin, Inequalities: theory of majorization and its applications, Academic Press, Serie Math. in Science and Engineering 143 (1979)

[13] L. Nirenberg, On elliptic partial differential equations (Lecture II), Ann. Sc. Norm. Sup. Pisa 13 (1959), 123-131.

[14] R.D. Nussbaum, Periodic solutions of nonlinear autonomous functional differential equations, Functional differential equations and approximation of fixed points, Lecture Notes in Mathematics 730, Springer-Verlag (1979), 283-325.

$\mathrm{RR} \mathrm{n}^{\circ} 3443$ 
[15] H. Peters, Chaotic behaviour of nonlinear differential-delay equations, Nonlinear Analysis, Theory, Methods and Applications, vol. 7, no 12 (1983), 1315-1334.

[16] I. Schur, Über eine Klasse von Mittelbildungen mit Anwendungen die Determinanten Theorie Sitzungsber. Berlin. Math. Gesellschaft 22 (1923), 9-20 (Isaie Schur collected works (A. Brauer and H. Rohrbach, eds.) Vol. II, Springer-Verlag, Berlin (1973), 416427)

[17] E. Shustin, Super-high-frequency oscillations in a discontinuous dynamic system with time delay, Israel J. of Mathematics, 90 (1995), 199-219.

[18] E. Shustin, E. Fridman, L. Fridman, Stable oscillations in a discontinuous delay system of the second order, Proc. Int. Conf. on Differential equations and dynamical systems, Springfield (Missouri), May 29-June 1, 1996.

[19] M. Tomić, Théorème de Gauss relatif au centre de gravité et son application, Bull. Soc. Math. Phys. Serbie 1 (1949), 31-40

[20] H. Weyl, Inequalities between two kinds of eigenvalues of a linear transformation, Proc. Nat. Acad. Sci. U.S.A. 35 (1949), 408-411 
Unit`e de recherche INRIA Lorraine, Technopôle de Nancy-Brabois, Campus scientifique, 615 rue du Jardin Botanique, BP 101, 54600 VILLERS LÈS NANCY

Unit'e de recherche INRIA Rennes, Irisa, Campus universitaire de Beaulieu, 35042 RENNES Cedex Unit'e de recherche INRIA Rhône-Alpes, 655, avenue de l'Europe, 38330 MONTBONNOT ST MARTIN Unit'e de recherche INRIA Rocquencourt, Domaine de Voluceau, Rocquencourt, BP 105, 78153 LE CHESNAY Cedex Unit'e de recherche INRIA Sophia-Antipolis, 2004 route des Lucioles, BP 93, 06902 SOPHIA-ANTIPOLIS Cedex

Éditeur

INRIA, Domaine de Voluceau, Rocquencourt, BP 105, 78153 LE CHESNAY Cedex (France) http://www.inria.fr ISSN 0249-6399 